\title{
High Pressure Flames with Multicomponent Transport
}

\author{
Vincent GIOVANGIGLI* ${ }^{\star}$, Lionel MATUSZEWSKI ${ }^{\dagger}$, and Pierre GAILLARD ${ }^{\dagger}$ \\ ${ }^{\star}$ CMAP-CNRS, Ecole Polytechnique, 91128 Palaiseau France \\ ${ }^{\dagger}$ ONERA, Chemin de la Hunière, Palaiseau France
}

\begin{abstract}
The thermodynamic formulation and the traditional formulation of multicomponent transport fluxes in high pressure fluids are discussed. The impact of high pressure transport models on mixing layers, premixed plane flames and strained diffusion flames is then investigated. Multicomponent fluxes in diffuse-interface transcritical diffusion flames are further addressed.
\end{abstract}

\section{Introduction}

Supercritical cryogenic combustion is often encountered in rocket engines. This has motivated modeling and numerical simulation of supercritical mixing and combustion in laminar flows $[1,2,3,4,5,6,7,8,9]$ and turbulent flows $[10,11,12,13,14,15,16,17]$ as well as model rocket combustor experiments [18, 19, 20, 21]. At such high-pressure and low temperature conditions, however, multicomponent transport phenomena is highly complex and nonidealities may influence the structure and dynamics of mixing zones as well as flames. With such an incentive we review and discuss in this chapter high pressure multicomponent transport models and their impact on the structure of mixing layers and supercritical flames.

The equations governing nonideal gas mixtures and the expression of multicomponent fluxes in terms of chemical potential gradients and temperature gradients - the so called thermodynamic form — with proper symmetry of the coefficients have first been derived in the framework of the thermodynamic of irreversible processes by Meixner [22, 23] and Prigogine [24]. In such a framework, the fluxes are obtained after expressing entropy production as a quadratic form, writting the fluxes as linear expressions of the gradients, and using Onsager symmetry relations [25]. An elegant presentation of this theory and of the historical developments is given in the book of de Groot and Mazur [26]. Similar expressions had been written previously by Eckart [27] but only for ideal gas mixtures and without symmetry properties of the transport coefficients.

Such equations governing dense gases have also been obtained from statistical mechanics. The equations governing dense single gases have been obtained by Irwing and Kirkwood [28] and the equations for dense gas mixtures by Bearman and Kirkwood [29] and Mori [30]. The transport fluxes have been expressed in terms of averages over molecular distribution functions and the thermodynamic form of transport fluxes has been rederived by Bearman and Kirkwood [29] and Mori [30].

The governing equations and the multicomponent fluxes have also been obtained by Keizer [31] from statistical thermodynamics. Keizer has developed an extended statistical theory of nonequilibrium processes which includes nonlinear effects and is applicable close to as well as far from equilibrium. In this fluctuation dissipation theory, elementary molecular processes manifest themselves as random changes in the extensive variables characterizing a system [31]. The importance of Keizer's results in statistical thermodynamics for high pressure combustion modeling has been emphasized in particular by Bellan and coworkers $[2,11,14]$.

The kinetic theory of gases is well understood for dilute gas mixtures but the resulting equation of state is that of perfect gases [32, 33, 34, 35, 36, 37]. A kinetic theory of dense gases has first been given by Enskog for rigid spheres and extended by Thorne to binary mixtures [34, 35]. The advantage of the rigid sphere model is that collisions are instantaneous so that the probability of simultaneous multiple encounters is negligible. The principal transport mechanism in dense gases is then collisional transfer at variance with dilute gases where it is molecular flow. It has been found, however, that the results of the Enskog-Thorne theory - and of its straightforward extensions - are not compatible with the thermodynamics of irreversible processes [38]. A modified form of Enskog equation has then been 
introduced by Van Beijeren and Ernst [39] and the resulting dense fluid gas mixture kinetic theory [40] has been shown to be compatible with the thermodynamics of irreversible processes. The corresponding Chapman-Enskog procedure has been performed by Kurochkin et al. [41]. More general theories of dense gases are based on multiple velocity distribution functions and on the BBGKY hierarchy of equations $[34,35]$. Formal expressions have notably been derived for a single gas, assuming that the two-particle distribution function is time-independent [35]. Nevertheless, to the authors' knowledge, a complete and fully satisfactory kinetic theory of dense polyatomic reactive gas mixtures is still missing.

The previously mentioned theories have yielded the conservation equations and the structure of transport fluxes that are used in this work as well as compatibility with the second principle of thermodynamics. On the other hand, in the absence of a satisfactory kinetic theory of dense mixtures, thermodynamics are typically built from accurate approximate state laws assuming a Gibbsian structure and compatibility with perfect gases at low density $[1,2,3,4,5,6,42]$. The thermodynamics used in this work is based on the Soave-Redlich-Kwong cubic equation of state [43, 44, 45, 46, 47, 48, 49]. The corresponding chemical production rates are derived from statistical mechanics, statistical thermodynamics, or the kinetic theory of gases [50, 51, 31, 52]. An important consequence of such nonideal thermodynamics is the presence of thermodynamic instabilities that may be of thermal, mechanical or chemical type [5]. In the absence of a satisfactory kinetic theory of dense mixtures, the shear viscosity and the thermal conductivity are also evaluated from high pressure correlations [53, 54]. Binary diffusion coefficients are obtained from the kinetic theory of dense mixtures of hard spheres [40, 41], allowing to take into account steric effects. The multicomponent diffusion coefficients are then obtained as approximate pseudo inverses of the Stefan-Maxwell matrix [5].

The natural thermodynamic form of multicomponent diffusion and heat fluxes is obtained in terms of chemical potential gradients and thus automatically involves nonideal effects in an implicit fashion [26, $31,40,5]$. Such a thermodynamic form also naturally arises in the mathematical theory of high pressure fluids [55] and is also useful in order to evaluate multicomponent fluxes in all pressure transcritical flame models in the presence of mechanical thermodynamic stability limits. On the other hand, the traditional form of transport fluxes may also be obtained by introducing the proper high pressure coefficients as well as the species diffusion driving forces. Nonidealities are then made explicit in the diffusion driving forces by developing the derivatives of chemical potentials with respect to mole or mass fractions at constant temperature. Nonidealities in diffusion driving forces have been considered in particular by Okong'o and Bellan [2], Oefelein [10], Bellan [11] and Giovangigli, Matuszewski, and Dupoirieux [5]. Transport nonidealities are notably strong in cold zones of premixed flames and prevent unrealistic diffusion from dense fresh reactants into flame fronts [5]. Nonideal diffusion also has a strong influence on the structure of cold jets near chemical thermodynamic stability limits between two fluids $[6,8]$. Nonideal diffusion has also been considered with diffuse interface transcritical flames [7].

The high pressure fluid model and the multicomponent fluxes are detailed in Section 2 and the structure of binary mixing layers is discussed in Section 3. The impact of high pressure transport models on plane and strained flames is adressed in Sections 4 and 5. Finally the situation of transcritical diffusion flames is investigated in Section 6.

\section{Nonideal fluids}

The equations governing high pressure fluids may be derived from the thermodynamics of irreversible processes [22, 23, 24, 26], statistical thermodynamics [31], statistical mechanics [28, 29, 30], as well as the kinetic theory of dense gases [40, 41].

\subsection{Conservation equations}

The equations expressing the conservation of species mass, momentum and energy in a dense fluid may be written in the form

$$
\begin{aligned}
& \partial_{t} \rho_{i}+\boldsymbol{\nabla} \cdot\left(\rho_{i} \boldsymbol{v}\right)+\boldsymbol{\nabla} \cdot \mathcal{J}_{i}=m_{i} \omega_{i}, \quad i \in \mathfrak{S}, \\
& \partial_{t}(\rho \boldsymbol{v})+\boldsymbol{\nabla} \cdot(\rho \boldsymbol{v} \otimes \boldsymbol{v}+p \boldsymbol{I})+\boldsymbol{\nabla} \cdot \boldsymbol{\Pi}=0, \\
& \partial_{t}\left(\rho\left(e+\frac{1}{2}|\boldsymbol{v}|^{2}\right)\right)+\boldsymbol{\nabla} \cdot\left(\rho\left(e+\frac{1}{2}|\boldsymbol{v}|^{2}\right) \boldsymbol{v}+p \boldsymbol{v}\right)+\boldsymbol{\nabla} \cdot\left(\mathcal{J}_{\mathrm{e}}+\boldsymbol{\Pi} \cdot \boldsymbol{v}\right)=0,
\end{aligned}
$$

where $\partial_{t}$ denotes the time derivative operator, $\boldsymbol{\nabla}$ the spatial differential operator, $\rho_{i}$ the mass density of the $i$ th species, $\mathcal{J}_{i}$ the diffusion flux of the $i$ th species, $m_{i}$ the molar mass of the $i$ th species, $\omega_{i}$ the molar production rate of the $i$ th species, $\mathfrak{S}=\{1, \ldots, n\}$ the species indexing set, $n$ the number of 
species, $\rho=\sum_{i \in \mathfrak{S}} \rho_{i}$ the mass density, $\boldsymbol{v}$ the fluid velocity, $p$ the pressure, $\boldsymbol{I}$ the identity tensor, $\boldsymbol{\Pi}$ the viscous tensor, $e$ the energy per unit mass of the mixture, and $\mathcal{J}_{\text {e }}$ the heat flux. It is assumed for the sake of simplicity that there are no forces acting on the species.

These equations need to be completed by relations expressing the thermodynamic properties $e$ and $p$, the chemical production rates $\omega_{i}, i \in \mathfrak{S}$, and the multicomponent fluxes $\mathcal{J}_{i}, i \in \mathfrak{S}, \boldsymbol{\Pi}$ and $\mathcal{J}_{\mathrm{e}}$. One may further define the pressure tensor $\mathcal{P}=p \boldsymbol{I}+\boldsymbol{\Pi}$ as well as the energy flux $\mathcal{Q}=\mathcal{J}_{\mathrm{e}}+\mathcal{P} \cdot \boldsymbol{v}$. The total momentum flux matrix is then $\rho \boldsymbol{v} \otimes \boldsymbol{v}+\mathcal{P}$ and the total energy vector flux $\rho \boldsymbol{v}\left(e+\frac{1}{2}|\boldsymbol{v}|^{2}\right)+\mathcal{Q}$. These quantities $\mathcal{P}$ and $\mathcal{Q}$ will be convenient in order to denote transport fluxes for fluid models with diffuse interfaces.

\subsection{Thermodynamics}

We will use in these notes the Soave-Redlich-Kwong equation of state [43, 44]

$$
p=\sum_{i \in \mathfrak{S}} \frac{\mathrm{y}_{i}}{m_{i}} \frac{R T}{v-b}-\frac{a}{v(v+b)},
$$

where $R$ denotes the perfect gas constant, $\mathrm{y}_{i}=\rho_{i} / \rho$ the mass fraction of the $i$ th species, $v=1 / \rho$ the volume per unit mass, $a$ the massic attractive parameter, and $b$ the massic repulsive parameter. The parameters of the SRK equation of state $a(\mathrm{y}, T)$ and $b(\mathrm{y})$ may be evaluated from the Van der Waals mixing rules $a=\sum_{i, j \in \mathfrak{S}} \mathrm{y}_{i} \mathrm{y}_{j} \alpha_{i} \alpha_{j}$ and $b=\sum_{i \in \mathfrak{S}} \mathrm{y}_{i} b_{i}$ where y denotes the vector of species mass fractions $\mathrm{y}=\left(\mathrm{y}_{1}, \ldots, \mathrm{y}_{n}\right)^{t}$. The pure-component parameters $\alpha_{i}(T)$ and $b_{i}$ are obtained from critical point data or Lennard-Jones potential parameters [5]. The validity of this equation of state (4) and of the mixing rules have been carefully studied by comparison with NIST data by Congiunti et al. [45] and with results of Monte Carlo simulations by Colonna and Silva [46] and Cañas-Marín et al. [47, 48]. The SRK equation of state yields a good representation of dense to dilute fluids and still allows a fast inversion by using Cardan's formula.

With the pressure law (4) given, there exists a unique associated Gibbsian thermodynamics compatible at low densities with that of perfect gases [49,42]. The corresponding specific energy $e$ and entropy $s$ per unit mass are found in the form

$$
\begin{aligned}
e & =\sum_{i \in \mathfrak{S}} \mathrm{y}_{i}\left(e_{i}^{\mathrm{st}}+\int_{T^{\mathrm{st}}}^{T} c_{\mathrm{v} i}^{\mathrm{PG}}(\theta) \mathrm{d} \theta\right)+\frac{T \partial_{T} a-a}{b} \log \left(1+\frac{b}{v}\right), \\
s & =\sum_{i \in \mathfrak{S}} \mathrm{y}_{i}\left(s_{i}^{\mathrm{st}}+\int_{T^{\mathrm{st}}}^{T} \frac{c_{\mathrm{p} i}^{\mathrm{PG}}(\theta)}{\theta} \mathrm{d} \theta\right)-\sum_{i \in \mathfrak{S}} \mathrm{y}_{i} \frac{R}{m_{i}} \log \left(\frac{\mathrm{y}_{i} R T}{m_{i}(v-b) p^{\mathrm{st}}}\right)+\frac{\partial_{T} a}{b} \log \left(1+\frac{b}{v}\right),
\end{aligned}
$$

where $e_{i}^{\text {st }}$ is the formation energy of the $i$ th species at the standard temperature $T^{\text {st }}, s_{i}^{\text {st }}$ the formation entropy of the $i$ th species at the standard temperature $T^{\text {st }}$ and standard pressure $p^{\text {st }}, c_{\mathrm{v} i}^{\text {PG }}$ the perfectgas specific heat at constant volume of the $i$ th species and $c_{\mathrm{p} i}^{\mathrm{PG}}$ the perfect-gas specific heat at constant pressure of the $i$ th species. The enthalpy of the mixture $h$ as well as the Gibbs function $g$ per unit mass may next be obtained from $h=e+p v$ and $g=e+p v-T s$ respectively and for any thermodynamic function $\varphi \in\{e, s, h, g\}$ the corresponding species densities are obtained from $\varphi_{i}=\left(\partial_{\mathrm{y}_{i}} \varphi\right)_{p, T, \mathrm{y}_{j \neq i}}$. We will use in particular in the following the species enthalpies $h_{i}, i \in \mathfrak{S}$, the species Gibbs functions $g_{i}$, $i \in \mathfrak{S}$, as well as the chemical potentials $\mu_{i}=m_{i} g_{i} / R T, i \in \mathfrak{S}$,

The entropy of a stable isolated homogeneous system should be a concave function of its thermodynamic variables $w=\left(v, \mathrm{y}_{1}, \ldots, \mathrm{y}_{n}, e\right)^{t}$. Whenever it is not the case, there exists an unstable domain where the system loses its homogeneity and splits between two or more phases in order to reach equilibrium. The Hessian matrix $\partial_{w w}^{2} s$ must therefore be negative semi-definite with nullspace $N\left(\partial_{w w}^{2} s\right)=\mathbb{R} w$ associated with homogeneity [42]. One may establish that thermodynamic stability holds, that is, $\partial_{w w}^{2} s \leq 0$ with $N\left(\partial_{w w}^{2} s\right)=\mathbb{R} w$, if and only if the following properties hold

$$
\left(\partial_{T} e\right)_{v, \mathrm{y}}>0, \quad\left(\partial_{v} p\right)_{T, \mathrm{y}}<0, \quad\left(\partial_{\mathrm{yy}}^{2} g\right)_{T, p} \geq 0, \quad N\left(\left(\partial_{\mathrm{yy}}^{2} g\right)_{T, p}\right)=\mathbb{R} \mathbf{y}
$$

The first condition $\left(\partial_{T} e\right)_{v, y}>0$ is the thermal stability condition that generally holds with the SRK equation of state [42]. The second condition $\left(\partial_{v} p\right)_{T, \mathrm{y}}<0$ is the mechanical stability condition associated with liquid/vapor phase changes in single species fluids. The third and fourth conditions form the chemical stability condition and state that the Hessian matrix of the Gibbs function per unit mass $\mathcal{G}=\left(\partial_{\mathrm{yy}}^{2} g\right)_{T, p}$ should be positive semi-definite with nullspace $N(\mathcal{G})=\mathbb{R} y$ from homogeneity. The 
combined mechanical and chemical conditions are also equivalent to the property that the Hessian matrix at constant volume $\left(\partial_{y y}^{2} g\right)_{T, v}$ is positive definite [42].

In order to investigate the mathematical quality of a fluid model, it is notably required to derive a balance equation for the entropy $s$ and next to establish that entropy production is nonnegative. Using Gibb's relation $T \mathbb{D} s=\mathbb{D} e+p \mathbb{D} v-\sum_{k \in \mathfrak{S}} g_{k} \mathbb{D}_{k}$ where $\mathbb{D}$ denotes the total differential operator, and the governing equations (1)-(3), the entropy governing equation is found in the form

$$
\begin{array}{r}
\partial_{t}(\rho s)+\nabla \cdot(\rho \boldsymbol{v} s)+\boldsymbol{\nabla} \cdot\left(-\sum_{i \in \mathfrak{S}} \frac{g_{i}}{T} \mathcal{J}_{i}+\frac{1}{T} \mathcal{J}_{\mathrm{e}}\right)=-\sum_{i \in \mathfrak{S}} \boldsymbol{\nabla}\left(\frac{g_{i}}{T}\right) \cdot \mathcal{J}_{i}+\boldsymbol{\nabla}\left(\frac{1}{T}\right) \cdot \mathcal{J}_{\mathrm{e}} \\
-\frac{\boldsymbol{\Pi}: \boldsymbol{\nabla} \boldsymbol{v}}{T}-\sum_{i \in \mathfrak{S}} \frac{g_{i} m_{i} \omega_{i}}{T}
\end{array}
$$

The expressions for chemical productions rates $\omega_{i}, i \in \mathfrak{S}$, diffusion fluxes $\mathcal{J}_{i}, i \in \mathfrak{S}$, heat flux $\mathcal{J}_{\mathrm{e}}$, and viscous tensor $\boldsymbol{\Pi}$, have thus to ensure that entropy production in the right hand side of (8) is nonnegative.

\subsection{Chemical production rates}

An arbitrary complex reaction mechanism with $n^{r}$ reactions involving $n$ species may be written symbolically

$$
\sum_{i \in \mathfrak{S}} \nu_{i j}^{\mathrm{f}} \mathfrak{M}_{i} \rightleftarrows \sum_{i \in \mathfrak{S}} \nu_{i j}^{\mathrm{b}} \mathfrak{M}_{i}, \quad j \in \mathfrak{R},
$$

where $\nu_{i j}^{\mathrm{f}}$ and $\nu_{i j}^{\mathrm{b}}$ denote the forward and backward stoichiometric coefficients of the $i$ th species in the $j$ th reaction, $\mathfrak{M}_{i}$ the symbol of the $i$ th species, and $\mathfrak{R}=\left\{1, \ldots, n^{r}\right\}$ the chemical reaction indexing set. Denoting by $\nu_{j}^{\mathrm{b}}=\left(\nu_{1 j}^{\mathrm{b}}, \ldots, \nu_{n j}^{\mathrm{b}}\right)^{t}$ and $\nu_{j}^{\mathrm{f}}=\left(\nu_{1 j}^{\mathrm{f}}, \ldots, \nu_{n j}^{\mathrm{f}}\right)^{t}$ the forward and backward reaction vectors, the molar production rate vector $\omega=\left(\omega_{1}, \ldots, \omega_{n}\right)^{t}$ is then given by

$$
\omega=\sum_{j \in \mathfrak{R}}\left(\nu_{j}^{\mathrm{b}}-\nu_{j}^{\mathrm{f}}\right) \tau_{j},
$$

where $\tau_{j}$ denotes the rate of progress of the $j$ th reaction. The proper form for the rate of progress $\tau_{j}$ is deduced from statistical thermodynamics as well as the kinetic theory of gases $[50,51,31,52]$

$$
\tau_{j}=\mathcal{K}_{j}\left(\exp \left\langle\nu_{j}^{\mathrm{f}}, \mu\right\rangle-\exp \left\langle\nu_{j}^{\mathrm{b}}, \mu\right\rangle\right)
$$

where $\mathcal{K}_{j}>0$ denotes the symmetric reaction constant of the $j$ th reaction, $\langle a, b\rangle$ the Euclidean product between vectors $a$ and $b, \mu=\left(\mu_{1}, \ldots, \mu_{n}\right)^{t}$ the vector of chemical potentials, $\mu_{i}=m_{i} g_{i} / R T, i \in \mathfrak{S}$, the species molar dimensionless chemical potentials, and $g_{i}, i \in \mathfrak{S}$, the species Gibbs functions. These rates (11) coincide with the ideal gas rates in the perfect gas limit and are compatible with traditional nonidealities used to estimate equilibrium constants. These rates can further be written in the form of the law of mass action by using the species affinities [5, 42].

Entropy production associated with chemistry may be written $-\sum_{i \in \mathfrak{S}} g_{i} m_{i} \omega_{i} / T=-R\langle\mu, \omega\rangle$ and using the expression of the rates of progress (11) yields

$$
-\sum_{i \in \mathfrak{S}} \frac{g_{i} m_{i} \omega_{i}}{T}=R \sum_{j \in \mathfrak{R}} \mathcal{K}_{j}\left(\left\langle\nu_{j}^{\mathrm{f}}, \mu\right\rangle-\left\langle\nu_{j}^{\mathrm{b}}, \mu\right\rangle\right)\left(\exp \left\langle\nu_{j}^{\mathrm{f}}, \mu\right\rangle-\exp \left\langle\nu_{j}^{\mathrm{b}}, \mu\right\rangle\right) .
$$

Therefore, entropy production due to chemical reactions is a sum of nonnegative terms and only vanishes at chemical equilibrium, i.e., when $\left\langle\nu_{j}^{\mathrm{f}}, \mu\right\rangle=\left\langle\nu_{j}^{\mathrm{b}}, \mu\right\rangle$, for all reactions $j \in \mathfrak{R}[52,42]$. This expression of entropy production due to chemistry (12) may be also interpreted as a macroscopic analog of Boltzmann $\mathrm{H}$ theorem involving only reactive collisions.

\subsection{Thermodynamic form for transport fluxes}

The heat and mass transport fluxes in a dense fluid mixture may be written in their natural thermodynamic form [23, 24, 26, 29, 31, 40, 41]

$$
\begin{aligned}
& \mathcal{J}_{i}=-\sum_{j \in \mathfrak{S}} L_{i j} \nabla\left(\frac{g_{j}}{T}\right)-L_{i \mathrm{e}} \nabla\left(-\frac{1}{T}\right), \quad i \in \mathfrak{S} \\
& \mathcal{J}_{\mathrm{e}}=-\sum_{j \in \mathfrak{S}} L_{\mathrm{e} j} \nabla\left(\frac{g_{j}}{T}\right)-L_{\mathrm{ee}} \boldsymbol{\nabla}\left(-\frac{1}{T}\right),
\end{aligned}
$$


where $L_{i j}, i, j \in \mathfrak{S} \cup\{\mathrm{e}\}$, are the mass and heat transport coefficients. The matrix $L=\left(L_{i j}\right)_{i, j \in \mathfrak{S} \cup\{\mathrm{e}\}}$ is symmetric positive semi-definite with nullspace $N(L)=\mathbb{R} \mathcal{U}$, where $\mathcal{U}=(1, \cdots, 1,0)^{t}[31,55]$. This thermodynamic form $(13)(14)$ naturally arises from the thermodynamics of irreversible processes [26] as well as from statistical thermodynamics [31]. This form of the transport fluxes also naturally arises in the symmetrized form of the system of conservation laws (1)(3). Such symmetrized forms play a key role in the mathematical analysis of multicomponent fluids $[52,55]$ and involve the entropic variable $[52,55]$

$$
\mathrm{v}=\left(\frac{g_{1}}{T}, \ldots, \frac{g_{n}}{T}, \frac{-1}{T}\right)^{t}=R\left(\frac{\mu_{1}}{m_{1}}, \ldots, \frac{\mu_{n}}{m_{n}}, \frac{-1}{R T}\right)^{t}
$$

Letting then for convenience $\mathcal{J}=\left(\mathcal{J}_{1}, \ldots, \mathcal{J}_{n}, \mathcal{J}_{\mathrm{e}}\right)^{t}$ and $\nabla \mathrm{v}=\left(\boldsymbol{\nabla} \mathrm{v}_{1}, \ldots, \nabla \mathrm{v}_{n}, \boldsymbol{\nabla} \mathrm{v}_{\mathrm{e}}\right)^{t}$ we may rewrite (13)(14) in the form

$$
\mathcal{J}=-L \nabla \vee
$$

Entropy production associated with macroscopic gradients of concentrations and temperature in (8) may then be written

$$
-\sum_{i \in \mathfrak{S}} \nabla\left(\frac{g_{i}}{T}\right) \cdot \mathcal{J}_{i}+\nabla\left(\frac{1}{T}\right) \cdot \mathcal{J}_{\mathrm{e}}=\sum_{i, j \in \mathfrak{S}} L_{i j} \nabla \mathrm{v}_{i} \cdot \nabla \mathrm{v}_{j},
$$

and is therefore nonnegative since the matrix $L$ is symmetric positive semi-definite. The thermodynamic form $(13)(14)$ thus guarantee that entropy production due to molecular heat and mass transfer is nonnegative. These relations $(13)(14)$ will also be important in the modeling of diffuse interface transcritical flames in order to avoid flux singularities at mechanical thermodynamic stability limits.

On the other hand, the viscous tensor $\boldsymbol{\Pi}$ may be written in the form $[23,24,26,29,31,40,41]$

$$
\boldsymbol{\Pi}=-\mathfrak{v} \boldsymbol{\nabla} \cdot \boldsymbol{v} \boldsymbol{I}-\eta\left(\boldsymbol{\nabla} \boldsymbol{v}+\boldsymbol{\nabla} \boldsymbol{v}^{t}-\frac{2}{3} \boldsymbol{\nabla} \cdot \boldsymbol{v} \boldsymbol{I}\right)
$$

where $\mathfrak{v}$ denotes the volume viscosity and $\eta$ the shear viscosity. The entropy production due to viscous effects in (8) may then be rewritten after some algebra

$$
-\frac{\boldsymbol{\Pi}: \boldsymbol{\nabla} \boldsymbol{v}}{T}=\frac{\mathfrak{v}}{T}(\boldsymbol{\nabla} \cdot \boldsymbol{v})^{2}+\frac{\eta}{2 T}\left|\boldsymbol{\nabla} \boldsymbol{v}+\boldsymbol{\nabla} \boldsymbol{v}^{t}-\frac{2}{3}(\boldsymbol{\nabla} \cdot \boldsymbol{v})^{2} \boldsymbol{I}\right|^{2}
$$

and is thus nonnegative since $\mathfrak{v} \geq 0$ and $\eta>0$.

\subsection{Traditional form for transport fluxes}

The multicomponent fluxes (13)(14) may be written in a more traditional form upon introducing the species diffusion driving forces $\boldsymbol{d}_{j}, j \in \mathfrak{S}$, and the proper high pressure transport coefficients. The species diffusion driving forces are defined by

$$
\boldsymbol{d}_{j}=\mathrm{x}_{j}\left(\nabla \mu_{j}\right)_{T}=\mathrm{x}_{j} \nabla \mu_{j}+\frac{\mathrm{x}_{j} m_{j} h_{j}}{R T^{2}} \nabla T, \quad j \in \mathfrak{S},
$$

where $x_{j}$ denotes the mole fraction of the $j$ th species and $\left(\nabla \mu_{j}\right)_{T}$ the gradient of the chemical potential $\mu_{j}$ with the temperature $T$ held constant and $\mu_{j}$ written as a function of temperature, pressure and mole fractions $\mu_{j}\left(T, p, \mathrm{x}_{1}, \ldots, \mathrm{x}_{n}\right)$ or equivalently with mass fractions $\mu_{j}\left(T, p, \mathrm{y}_{1}, \ldots, \mathrm{y}_{n}\right)$. The diffusion driving force may be written in particular

$$
\boldsymbol{d}_{j}=\frac{\mathrm{x}_{j} m_{j} v_{j}}{R T} \nabla p+\sum_{l \in \mathfrak{S}} \Gamma_{j l} \nabla \mathrm{x}_{l}, \quad j \in \mathfrak{S},
$$

where $v_{j}, j \in \mathfrak{S}$, denote the species partial volume per unit mass of the $j$ th species, $\Gamma_{j l}$ the thermodynamic coefficients $\Gamma_{j l}=\mathrm{x}_{j}\left(\partial_{x_{l}} \mu_{j}\right)_{T, p, x_{k}}, j, l \in \mathfrak{S}$, and the matrix $\Gamma=\left(\Gamma_{i j}\right)_{i, j \in \mathfrak{S}}$ reduces to the identity matrix for ideal gases. High pressure coefficients may then be obtained from the modified matrix $\widetilde{L}$ defined by $\widetilde{L}=A^{t} L A$ with

$$
A=\left(\begin{array}{cccc} 
& & & -h_{1} \\
& I_{n} & & \vdots \\
& & & -h_{n} \\
0 & \cdots & 0 & 1
\end{array}\right) .
$$


The coefficients of the matrix $\widetilde{L}$ then directly yield the high pressure diffusion coefficients $D_{i j}, i, j \in \mathfrak{S}$, thermal diffusion coefficients $\theta_{i}, i \in \mathfrak{S}$, and partial thermal conductivity $\widehat{\lambda}$ with the expressions

$$
\frac{\widetilde{L}_{i j} R}{\rho \mathrm{y}_{i} \mathrm{y}_{j} m}=D_{i j}, \quad i, j \in \mathfrak{S}, \quad \frac{\widetilde{L}_{i \mathrm{e}}}{T}=\rho \mathrm{y}_{i} \theta_{i}, \quad i \in \mathfrak{S}, \quad \frac{\widetilde{L}_{\mathrm{ee}}}{T^{2}}=\hat{\lambda}
$$

where $m$ denotes the mean molecular weight of the mixture. Using these transport coefficients $(20)$ and the diffusion driving forces (17), the multicomponent fluxes (13)(14) are rewritten in the more traditional form $[32,34,35,37,52]$

$$
\begin{aligned}
\mathcal{J}_{i} & =-\sum_{j \in \mathfrak{S}} \rho \mathrm{y}_{i} D_{i j} \boldsymbol{d}_{j}-\rho \mathrm{y}_{i} \theta_{i} \boldsymbol{\nabla} \log T, \quad i \in \mathfrak{S}, \\
\mathcal{J}_{\mathrm{e}}-\sum_{i \in \mathfrak{S}} h_{i} \mathcal{J}_{i} & =-\frac{\rho R T}{m} \sum_{j \in \mathfrak{S}} \theta_{j} \boldsymbol{d}_{j}-\widehat{\lambda} \boldsymbol{\nabla} T .
\end{aligned}
$$

Even if (21) (22) is formally similar to a traditional formulation at lower pressure, it involves here high pressure transport coefficients. In addition, the traditional formulation (22) yields the reduced heat flux $\mathcal{J}_{\mathrm{e}}-\sum_{i \in \mathfrak{S}} h_{i} \mathcal{J}_{i}$ in contrast with the thermodynamic form (14) that directly yields the full heat flux

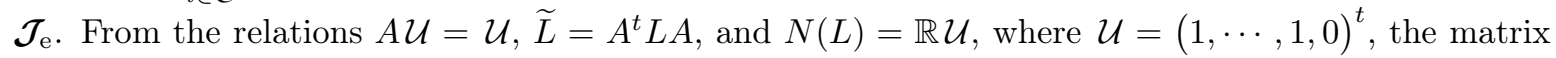
$\widetilde{L}$ is found to be symmetric positive semi-definite with nullspace $N(\widetilde{L})=\mathbb{R} \mathcal{U}$. As a consequence, the diffusion matrix $D=\left(D_{i j}\right)_{i, j \in \mathfrak{S}}$ is symmetric positive semi-definite with nullspace $N(D)=\mathbb{R}$ y where $\mathrm{y}=\left(\mathrm{y}_{1}, \ldots, \mathrm{y}_{n}\right)^{t}$ is the mass fraction vector, and the vector of Soret coefficients $\theta=\left(\theta_{1}, \ldots, \theta_{n}\right)^{t}$ is such that $\langle\theta, \mathrm{y}\rangle=0$. Since $D \mathrm{y}=0$ and $\langle\theta, \mathrm{y}\rangle=0$, the transport fluxes (21) (22) may also be rewritten in terms of the linearly dependent diffusion driving forces $\widetilde{\boldsymbol{d}}_{j}=\boldsymbol{d}_{j}-\mathrm{y}_{j} \sum_{l \in \mathfrak{S}} \boldsymbol{d}_{l}$ that sum up to zero.

In the low Mach number limit - where pressure gradients may be neglected - the diffusion driving forces (17) may further be simplified as $\boldsymbol{d}_{j}=\mathrm{x}_{j}\left(\boldsymbol{\nabla} \mu_{j}\right)_{T, p}, j \in \mathfrak{S}$, where $\left(\nabla \mu_{j}\right)_{T, p}$ denotes the spatial gradient of $\mu_{j}$ with $T$ and $p$ held constant. For perfect gases, after some algebra, using $\sum_{l \in \mathfrak{S}} \times_{l}=1$, one may also recover the usual formula $\boldsymbol{d}_{j}^{\mathrm{PG}}=\boldsymbol{\nabla} \mathrm{x}_{j}, j \in \mathfrak{S}$, for the diffusion driving forces [5].

An alternative form for mass and heat multicomponent fluxes - similar to that of dilute gases - is also obtained by introducing the generalized thermal diffusion ratios $\chi=\left(\chi_{1}, \ldots, \chi_{n}\right)^{t}$ and the thermal conductivity $\lambda$ with $[23,41,34]$

$$
\theta=D \chi, \quad\langle\chi, \mathrm{u}\rangle=0, \quad \lambda=\widehat{\lambda}-\frac{\rho R}{m}\langle D \chi, \chi\rangle,
$$

where $\mathbf{u}=(1, \ldots, 1)^{t}$ is the vector with units components. The transport linear system defining the thermal diffusion ratios $\theta=D \chi$ with the constraint $\langle\chi, \mathbf{u}\rangle=0$ is easily shown to be well posed [37] and the transport fluxes can then be written

$$
\begin{aligned}
\mathcal{J}_{i} & =-\sum_{j \in \mathfrak{S}} \rho \mathrm{y}_{i} D_{i j}\left(\boldsymbol{d}_{j}+\chi_{j} \boldsymbol{\nabla} \log T\right), \quad i \in \mathfrak{S}, \\
\mathcal{J}_{\mathrm{e}}-\sum_{i \in \mathfrak{S}} h_{i} \mathcal{J}_{i} & =\sum_{j \in \mathfrak{S}} \frac{R T}{m_{j}} \widetilde{\chi}_{j} \mathcal{J}_{j}-\lambda \nabla T
\end{aligned}
$$

where $\tilde{\chi}_{j}$ is the rescaled thermal diffusion ratio of the $j$ th species $\tilde{\chi}_{j}=\chi_{j} / x_{j}$. This formulation has been used in these notes - except for diffuse interface models where the thermodynamic form is mandatory - and is notably convenient for dilute gases since it is less expensive to evaluate $\lambda$ and $\chi$ than $\hat{\lambda}$ and $\theta[37]$.

\subsection{High pressure transport coefficients}

In order to take into account the influence of density on transport coefficients, the thermal conductivity $\lambda$ and the shear viscosity $\eta$ are evaluated as described by Chung et al. [53] and Ely and Hanley [54]. On the other hand, the species diffusion matrix $D$ is evaluated by solving Stefan-Maxwell type equations derived from the kinetic theory of dense gases by Kurochkin, Makarenko, and Tirskii [41] as well as from various experiments with liquid mixtures as comprehensively discussed by Taylor and Krishna [56]. Another variant is to use Grad's moments method as done by Harstad and Bellan [57]. However, Grad's moments method has been shown to be equivalent to the Chapman-Enskog method by Zhdanov 
in the framework of weakly ionized plasmas [58] and Harstad and Bellan have also obtained StefanMaxwell type equations for high pressure diffusion coefficients [57].

The Stefan-Maxwell matrix $\Delta=\left(\Delta_{i j}\right)_{i, j \in \mathfrak{S}}$ may be written

$$
\Delta_{i i}=\sum_{j \neq i} \frac{\mathrm{x}_{i} \mathrm{x}_{j}}{\mathcal{D}_{i j}}, \quad \Delta_{i j}=-\frac{\mathrm{x}_{i} \mathrm{x}_{j}}{\mathcal{D}_{i j}}, \quad i \neq j,
$$

where $\mathcal{D}_{i j}, i, j \in \mathfrak{S}, i \neq j$, are the species binary diffusion coefficients. Different expressions have been proposed in the literature for evaluating high pressure binary diffusion coefficients $\mathcal{D}_{i j}, i, j \in \mathfrak{S}, i \neq j$, notably by Wakeham [59], Takahashi [60], Harstad and Bellan [61], Leahy-Dios and Firoozbadi [62], and Matsuyama et al. [15]. The correlations of Takahashi have been used in particular by Ribert et al. [3], Pons et al. [4], Oefelein [10], and Zong and Yang [12]. Note that models and/or experimental correlations should clearly factorize the terms due to nonidealities [61,62], to prevent the risk of including them in the binary diffusion coefficients. In these notes, the binary diffusion coefficients have been obtained from the kinetic theory of dense mixtures of hard spheres developped by Beijeren and Ernst [40] and Kurochkin et al. [41] allowing to take into account steric effets in diffusive processes. These binary diffusion coefficients are evaluated in the form $\mathcal{D}_{i j}=\mathcal{D}_{i j}^{\text {PG }} / \Upsilon_{i j}, i, j \in \mathfrak{S}, i \neq j$, where $\mathcal{D}_{i j}^{\text {PG }}$ denotes the perfect gas binary diffusion coefficient given by the kinetic theory of dilute gases and $\Upsilon_{i j}$ is the factor

$$
\Upsilon_{i j}=1+\sum_{k \in \mathfrak{S}} \frac{\pi \mathfrak{n}_{k}}{12}\left(8\left(\sigma_{i k}^{3}+\sigma_{j k}^{3}\right)-6\left(\sigma_{i k}^{2}+\sigma_{j k}^{2}\right) \sigma_{i j}-3\left(\sigma_{i k}^{2}-\sigma_{j k}^{2}\right)^{2} \sigma_{i j}^{-1}+\sigma_{i j}^{3}\right),
$$

where $\sigma_{i j}$ denotes the collision diameter for the species pair $(i, j)$ and $\mathfrak{n}_{k}$ the number density of the $k$ th species.

The Stefan-Maxwell matrix $\Delta$ is symmetric positive semi-definite with nullspace $N(\Delta)=\mathbb{R} u$ where $\mathrm{u}=(1, \ldots, 1)^{t} \in \mathbb{R}^{n}$ and $2 \operatorname{diag}(\Delta)-\Delta$ is positive definite when $n \geq 3[37,52,63,64]$. From the kinetic theory of gases it is obtained that $\Delta D=I_{n}-\mathrm{y} \otimes \mathrm{u}$ with the mass constraint $D \mathrm{y}=0$. The diffusion matrix $D$ is shown to be the generalized inverse of $\Delta$ with prescribed nullspace $\mathbb{R} y$ and range $\mathrm{y}^{\perp}$ and for any $\alpha>0$ we have $D=(\Delta+\alpha \mathbf{y} \otimes \mathbf{y})^{-1}-(1 / \alpha) \mathbf{u} \otimes \mathbf{u}[37,52,63,64]$. Using the matrix $D$ is also equivalent to solving the Stefan-Maxwell equations for the diffusion velocities [37,63]. The resulting diffusion matrix $D$ is also fully populated, naturally taking account the interactions among species $[57,14]$. In order to accelerate the evaluation of the matrix $D$ from the Stefan-Maxwell matrix, we have used the theory of iterative algorithms for singular systems $[37,52,63,64,65,66,67,68]$. Introducing the splitting $\Delta=M-W$ where

$$
M=\operatorname{diag}\left(\frac{\Delta_{11}}{1-\mathrm{y}_{1}}, \ldots, \frac{\Delta_{n n}}{1-\mathrm{y}_{n}}\right)
$$

and letting $\mathcal{T}=M^{-1} W$ and $P=I_{n}-\mathbf{u} \otimes \mathrm{y}$, the following asymptotic expansion is shown to be convergent $[37,52,63,64]$

$$
D=\sum_{0 \leq j<\infty}(P \mathcal{T})^{j} P M^{-1} P^{t}
$$

Considering the first term $P M^{-1} P^{t}$, the matrix $M^{-1}$ corresponds to a generalization to high pressure of the Hirschfelder-Curtiss approximation and the projector $P$ to the addition of a species independent mass conservation corrector [63,67]. The next approximation of $D$ with two terms is more interesting since it is much more accurate and still yields $n^{2}$ coefficients within $\mathcal{O}\left(n^{2}\right)$ operations $[68,63,64]$. Harstad and Bellan have checked in particular that this two term approximation is accurate for high pressures [57]. Even the highly accurate three term approximation is interesting from a computational point of view since - thanks to symmetry - it is still approximately half the cost of a direct method using Cholesky algorithm with $n$ backsolves. These iterative algorithms have generally been found to be effective for fast and accurate evaluation of multicomponent diffusion matrices as well as transport coefficients $[63,64,65,66]$.

Finally, in order to evaluate the thermal diffusion ratios $\chi_{i}, i \in \mathfrak{S}$, we have generally used the limiting dilute gas value for $\chi_{i}, i \in \mathfrak{S}$. The main idea is that thermal diffusion will mainly influence the part of the flame which is sufficiently warm. Note that high pressure effects are still taken into account in the corresponding thermal diffusion coefficients $\theta=D \chi$ through the matrix $D$ which involves high pressure diffusion coefficients. 


\subsection{Numerical considerations}

The equations governing flame structures or mixing layers are discretized by using finite differences and solved on adaptive grids with the aid of Newton's method [69, 70]. Pseudo unsteady iterations may be used to bring an initial guess into the convergence domain of steady Newton's iteration. The grids are iteratively refined by adding discretization points wherever the solution gradients are too roughly represented.

Once a flame structure is obtained, it may be used as a starting point to compute solutions branches depending on a parameter. Branches are computed by means of continuation techniques [71] using global rezone adaptative griding that generates smooth grids. The solution branches are locally reparameterized by the most sensitive solution component hence allowing the computation of turning points and insuring that the solution is changing smoothly.

The evaluation of fluid properties such as chemical production rate, thermodynamic properties and transport coefficient is obtained with high pressure adapted versions of highly optimized thermochemistry and transport routines [72, 73, 74, 75].

When using the traditional form for diffusion velocities, it is also preferable to avoid double sums over the species arising from $\mathcal{J}_{i}=-\sum_{j \in \mathfrak{S}} \rho \mathrm{y}_{i} D_{i j} \boldsymbol{d}_{j}$ and the expansion in $\boldsymbol{d}_{j}$ of the derivatives of the chemical potentials $\left(\nabla \mu_{j}\right)_{T}$ or $\left(\nabla \mu_{j}\right)_{T, p}$ in terms of the species mole fraction gradients, keeping in mind that $\mu_{j}$ depend on all species for nonideal fluids. To this aim, the potential $\mu_{j}$ is decomposed in the form $\mu_{j}=\log \mathrm{x}_{j}+\mu_{j}^{\mathrm{sm}}$ where the smooth part $\mu_{j}^{\mathrm{sm}}$ may be evaluated explicitly. In the low Mach regime the diffusion driving force is then evaluated in the compact form

$$
\boldsymbol{d}_{j}=\mathrm{x}_{j}\left(\nabla \mu_{j}\right)_{T, p}=\nabla \mathrm{x}_{j}+\mathrm{x}_{j}\left(\nabla \mu_{j}^{\mathrm{sm}}\right)_{T, p}
$$

where the perfect gas contribution $\nabla \mathrm{x}_{j}$ is associated with the singular part $\log \mathrm{x}_{j}$ of $\mu_{j}$.

\section{$3 \quad$ Binary mixing layers}

The impact of nonidealities on the structure of binary mixing layers in the neighborhood of chemical thermodynamic stability limit is investigated [8].

\subsection{Binary diffusion matrices and fluxes}

For a binary mixture with $\mathfrak{S}=\{1,2\}$, the Stefan-Maxwell matrix (26) reduces to

$$
\Delta=\frac{\mathrm{x}_{1} \mathrm{x}_{2}}{\mathcal{D}_{12}}\left(\begin{array}{cc}
1 & -1 \\
-1 & 1
\end{array}\right)
$$

where $\mathcal{D}_{12}$ is the binary diffusion coefficient of the mixture pair. In this simplified situation, the mass constraint $D \mathrm{y}=0$ yields $\mathrm{y}_{1} D_{11}+\mathrm{y}_{2} D_{12}=0$ and $\mathrm{y}_{1} D_{21}+\mathrm{y}_{2} D_{22}=0$, and since $D_{12}=D_{21}$ by symmetry, all coefficients may be expressed in terms of $D_{12}$. From the Stefan-Maxwell relation $\Delta D=I_{2}-\mathrm{y} \otimes \mathrm{u}$, it is obtained after a little algebra that $D_{12}=-\mathcal{D}_{12} \frac{m_{1} m_{2}}{m^{2}}$. The multicomponent diffusion matrix is thus in the form

$$
D=\mathcal{D}_{12} \frac{m_{1} m_{2}}{m^{2}}\left(\begin{array}{cc}
\frac{\mathrm{y}_{2}}{\mathrm{y}_{1}} & -1 \\
-1 & \frac{\mathrm{y}_{1}}{\mathrm{y}_{2}}
\end{array}\right),
$$

and it is easily checked that $D$ is positive semi-definite with nullspace $\mathbb{R} y$ where $\mathrm{y}=\left(\mathrm{y}_{1}, \mathrm{y}_{2}\right)^{t}$.

On the other hand, the Hessian matrix $\mathcal{G}=\left(\partial_{\mathrm{yy}}^{2} g\right)_{T, p}$ of Gibbs function, where the derivations are performed with respect to mass fractions y while $T$ and $p$ are fixed, is closely associated with chemical thermodynamic stability as detailed in Section 2.2. The coefficients of the Hessian matrix $\mathcal{G}=\left(\mathcal{G}_{j l}\right)_{j, l \in \mathfrak{S}}$ are $\mathcal{G}_{j l}=\left(\partial_{\mathrm{y}_{l} \mathrm{y}_{j}}^{2} g\right)_{T, p}=\left(\partial_{\mathrm{y}_{l}} g_{j}\right)_{T, p}, j, l \in \mathfrak{S}$ and since the Gibbs function per unit mass $g$ is 1-homogeneous in terms of $\mathrm{y}-$ when $T$ and $p$ are constant-the derivatives $g_{i}, i \in \mathfrak{S}$, with respect to mass fractions are 0-homogeneous. From the Euler relation, the hessian matrix $\mathcal{G}$ is therefore such that $\mathcal{G} \mathrm{y}=0$ so that $\mathcal{G}_{11} \mathrm{y}_{1}+\mathcal{G}_{12} \mathrm{y}_{2}=0$ and $\mathcal{G}_{21} \mathrm{y}_{1}+\mathcal{G}_{22} \mathrm{y}_{2}=0$. Introducing the mass based coefficient

$$
\gamma=-\mathcal{G}_{12} \frac{m_{1} m_{2}}{m R T}=-\left(\partial_{\mathrm{y}_{1}} \mu_{2}\right)_{T, p} \frac{m_{1}}{m}=-\left(\partial_{\mathrm{y}_{2}} \mu_{1}\right)_{T, p} \frac{m_{2}}{m},
$$

we may write $\mathcal{G}$ in the form

$$
\mathcal{G}=\frac{m R T}{m_{1} m_{2}} \gamma\left(\begin{array}{cc}
\frac{\mathrm{y}_{2}}{\mathrm{y}_{1}} & -1 \\
-1 & \frac{\mathrm{y}_{1}}{\mathrm{y}_{2}}
\end{array}\right) .
$$


It is then easily established that $\mathcal{G} \geq 0$ is equivalent to $\gamma \geq 0$ and $N(\mathcal{G})=\mathbb{R y}$ is equivalent to $\gamma \neq 0$ using that $\langle\mathcal{G} z, z\rangle=\gamma m R T / m_{1} m_{2} \mathrm{y}_{1} \mathrm{y}_{2}$ where $z=(1,-1)^{t}$. Therefore thermodynamic stability holds if and only if $\gamma>0$ and a direct calculation yields that $\gamma=1$ for perfect gases.

We only consider in the following a low Mach fluid and the expression of the diffusion driving fluxes reduces to $\boldsymbol{d}_{j}=\mathrm{x}_{j}\left(\nabla \mu_{j}\right)_{T, p}, j \in \mathfrak{S}$. We will also neglect the Soret and Dufour effects since the mixing layers considered in this chapter only involve mild temperature gradients. The diffusion driving forces may thus be written $\boldsymbol{d}_{j}=\mathrm{x}_{j}\left(\nabla \mu_{j}\right)_{T, p}=\sum_{l \in \mathfrak{S}} \frac{\mathrm{y}_{j} m}{R T} \mathcal{G}_{j l} \nabla \mathrm{y}_{l}, j \in \mathfrak{S}$, and the fluxes then read

$$
\mathcal{J}_{i}=-\sum_{j, k \in \mathfrak{S}} \frac{\rho m}{R T} \mathrm{y}_{i} \mathrm{y}_{j} D_{i j} \mathcal{G}_{j k} \nabla \mathrm{y}_{k}, \quad i \in \mathfrak{S} .
$$

In our binary case it yields for the first species

$$
\mathcal{J}_{1}=-\rho \mathrm{y}_{1} D_{11} \frac{m \mathrm{y}_{1}}{R T}\left(\mathcal{G}_{11} \nabla \mathrm{y}_{1}+\mathcal{G}_{12} \nabla \mathrm{y}_{2}\right)-\rho \mathrm{y}_{1} D_{12} \frac{m \mathrm{y}_{2}}{R T}\left(\mathcal{G}_{21} \nabla \mathrm{y}_{1}+\mathcal{G}_{22} \nabla \mathrm{y}_{2}\right) .
$$

Using then $\mathrm{y}_{1}+\mathrm{y}_{2}=1, \nabla \mathrm{y}_{1}=-\nabla \mathrm{y}_{2}$, as well as the simplified expressions (30)(32) for $D$ and $\mathcal{G}$ it is found that

$$
\mathcal{J}_{1}=-\rho \mathcal{D}_{12} \gamma \mathrm{y}_{1}\left\{\frac{\mathrm{y}_{2}}{\mathrm{y}_{1}} \mathrm{y}_{1}\left(\frac{\mathrm{y}_{2}}{\mathrm{y}_{1}}+1\right)-\mathrm{y}_{2}\left(-1-\frac{\mathrm{y}_{1}}{\mathrm{y}_{2}}\right)\right\} \nabla \mathrm{y}_{1},
$$

and finally - after some algebra - that

$$
\mathcal{J}_{1}=-\rho \gamma \mathcal{D}_{12} \nabla \mathrm{y}_{1}
$$

This remarkably simple expression exactly reduces the matrix diffusion process involving the multicomponent diffusion matrix $D$ as well as the Hessian matrix $\mathcal{G}$ to a simple diagonal diffusion model provided that the fluxes are expressed in terms of mass fraction gradients and the coefficient $\gamma$ is a mass based coefficient. In such a binary situation, the quantity that is thermodynamically conjugated to $\mathrm{y}_{1}$ is $\mu_{1}-\mu_{2}$ keeping in mind that the relation $\nabla \mathrm{y}_{1}=-\nabla \mathrm{y}_{2}$ has been used. Similar expressions have also been presented in terms of molar based quantities [76, 77, 78, 79].

The expression (34) further shows how diffusion processes vanish at the chemical stability limit $\gamma=0$. When $\gamma$ is negative, there is then a spinodal decomposition between the two equilibrium states characterized by the bitangent condition. In this situation $\gamma<0$, higher order derivative terms from the second gradient theory of diffuse interfaces are required in order to stabilize mathematically the governing equations and in order to simulate numerically unmixing processes. When thermodynamic stability is lost, both hyperbolicity and parabolicity are indeed lost in the system of partial differential equations.

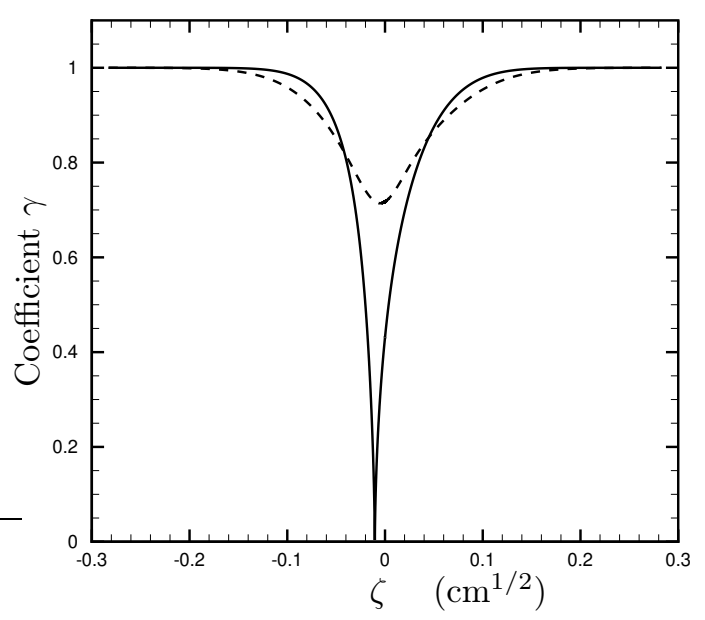

Figure 1: Coefficient $\gamma$ in a mixing layer at $\bar{p}=100 \mathrm{~atm} ; \boldsymbol{-}-T_{ \pm}=160 ;-T_{ \pm}=122 \mathrm{~K}$

\subsection{Simplified self-similar mixing layer equations}

We consider a steady, low-Mach, self-similar mixing layer between two species. The mixing layer is developing in the positive $x$ direction starting from $x=0$ where $x$ and $y$ denote the longitudinal and 


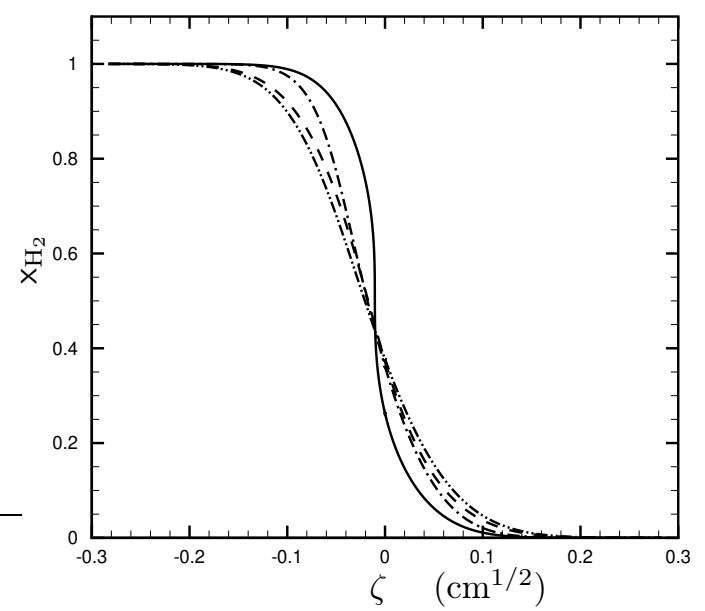

Figure 2: Hydrogen mole fraction in a mixing layer at $\bar{p}=100 \mathrm{~atm} ;-T_{ \pm}=122$ with nonideal diffusion; _. $T_{ \pm}=122$ with ideal diffusion; _ - $T_{ \pm}=160$ with nonideal diffusion; _... $T_{ \pm}=160$ with ideal diffusion

transverse coordinates with $\boldsymbol{x}=(x, y, z)^{t}$. The longitudinal and transverse velocity components are denoted by $u$ and $v$, respectively, so that $\boldsymbol{v}=(u, v, 0)^{t}$. The new simplified coordinates $\xi$ and $\zeta$ used in this work are defined by [8]

$$
\xi=x, \quad \zeta=y / x^{1 / 2}
$$

and the auxiliary pseudo velocity $w$ reads

$$
w=\sqrt{\xi} v-\frac{1}{2} \zeta u
$$

These coordinates (35) appear to be much simpler than traditional boundary layer type coordinates. For a self similar layer, the dependent unknowns $u, w, T, \mathrm{y}_{1}$ then only depend on $t$ and $\zeta$ whereas $v$ also depends on $\xi$ from $(36)$.

The resulting steady self-similar layer equations are then in the form [8]

$$
\begin{gathered}
\partial_{\zeta}(\rho w)+\frac{1}{2} \rho u=0 \\
\partial_{\zeta} \widetilde{p}=0 \\
\rho w \partial_{\zeta} u-\partial_{\zeta}\left(\eta \partial_{\zeta} u\right)=0 \\
\rho w \partial_{\zeta} \mathrm{y}_{1}-\partial_{\zeta}\left(\rho \mathcal{D}_{12} \gamma \partial_{\zeta} \mathrm{y}_{1}\right)=0 \\
\rho w \partial_{\zeta} h-\partial_{\zeta}\left(\lambda \partial_{\zeta} T+\rho\left(h_{1}-h_{2}\right) \gamma \mathcal{D}_{12} \partial_{\zeta} \mathrm{y}_{1}\right)=0
\end{gathered}
$$

where $\widetilde{p}$ denotes the perturbed pressure and $u, w, \rho, \mathrm{y}_{1}, h$ and $T$ only depend on $\zeta$. The pressure has been decomposed as usual in the form $p=\bar{p}+\widetilde{p}$ where $\bar{p}$ denotes the ambient pressure that is constant in space and $\widetilde{p}$ the fluid dynamic perturbation with the ratio $\widetilde{p} / \bar{p}$ of the order of the square of the Mach number [52]. Further assuming that the limiting longitudinal velocities $u( \pm \infty)$ are independent of $x$, it is found that $\partial_{\xi} \widetilde{p}=0$ and combining with (38) we obtain $\nabla \widetilde{p}=0$ so that $\widetilde{p}=0$ from boundary conditions and the pressure is constant $p=\bar{p}$.

The computational domain is typically taken in the form $\left[\mathrm{L}_{\zeta}^{-}, \mathrm{L}_{\zeta}^{+}\right]$and the boundary conditions are in the form

$$
\begin{array}{lll}
u\left(\mathrm{~L}_{\zeta}^{-}\right)=u_{-}, & T\left(\mathrm{~L}_{\zeta}^{-}\right)=T_{-}, & \mathrm{y}_{1}\left(\mathrm{~L}_{\zeta}^{-}\right)=1, \\
u\left(\mathrm{~L}_{\zeta}^{+}\right)=u_{+}, & T\left(\mathrm{~L}_{\zeta}^{+}\right)=T_{+}, & \mathrm{y}_{1}\left(\mathrm{~L}_{\zeta}^{+}\right)=0,
\end{array}
$$

and Ting boundary condition for the transverse velocity reads [80, 81]

$$
\rho_{-} u_{-} v_{-}+\rho_{+} u_{+} v_{+}=0 \text {. }
$$




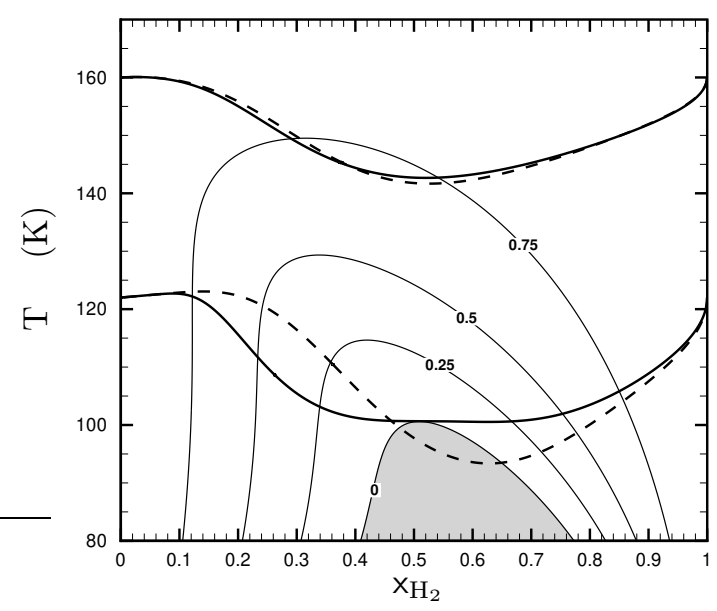

Figure 3: Phase diagram with mixing layer trajectories at $\bar{p}=100 \mathrm{~atm}, T_{ \pm}=160 \mathrm{~K}$ and $T_{ \pm}=122 \mathrm{~K}$; _ nonideal diffusion; _ _ ideal diffusion.

\subsection{Thermodynamic stability of $\mathrm{H}_{2}-\mathrm{N}_{2}$ mixtures}

Thermodynamic stability of fresh reactants is of fundamental importance for mixing layers as well as premixed cryogenic flames. An exhaustive study of thermodynamic stability for ternary mixtures $\mathrm{H}_{2}-\mathrm{O}_{2}-\mathrm{N}_{2}$ has been performed at various pressures using the thermodynamics built from the SRK equation of state [5, 42]. Similar results have been obtained at various pressures as long as they are above the critical pressures of $\mathrm{H}_{2}, \mathrm{O}_{2}$, and $\mathrm{N}_{2}$. In order to investigate the corresponding stability domain, we have located the points where the first eigenvalue of the matrix $\left(\partial_{\mathrm{yy}}^{2} g\right)_{T, v}$ is changing of sign by inspecting the zero values of its determinant. To this aim, we have used nonlinear solvers and continuation methods in order to generate the whole stability domain. The boundary of the stability domain are in general chemical stability limits that coincide with the mechanical stability limits in the asymptotic situation of pure fluids.

Verschoyle [82] and Eubanks [83] have investigated binary mixtures of $\mathrm{H}_{2}$ and $\mathrm{N}_{2}$ at high pressure and low temperature. A first important experimental result is that binary mixtures of $\mathrm{H}_{2}$ and $\mathrm{N}_{2}$ may not be thermodynamically stable at sufficiently high pressure and low temperature. In these situations, a mixture of $\mathrm{H}_{2}$ and $\mathrm{N}_{2}$ splits between a hydrogen-rich gaseous-like phase and a hydrogen-poor liquidlike phase [82, 83]. Detailed comparisons have been conducted between experimental results and numerical simulations of equilibrium states using the SRK equation of state [5, 42]. These simulations indicate that the instabilities are of chemical type and very good agreement has been obtained with experimental results [5].

\subsection{Impact of nonideal transport}

A cryogenic supercritical mixing layer between $\mathrm{H}_{2}$ and $\mathrm{N}_{2}$ is investigated and the species indexing set $\mathfrak{S}=\left\{\mathrm{H}_{2}, \mathrm{~N}_{2}\right\}$ is used for convenience. Hydrogen is injected on the left side $\mathrm{y}_{\mathrm{H}_{2}-}=1$, at temperature either $T_{-}=160 \mathrm{~K}$ or $T_{-}=122 \mathrm{~K}$ with longitudinal velocity $u_{-}=1 \mathrm{~cm} / \mathrm{sec}$, and the pressure is uniform at $\bar{p}=100 \mathrm{~atm}$. Nitrogen is on the right side $\mathrm{y}_{\mathrm{H}_{2}+}=0$, at either temperature $T_{+}=160 \mathrm{~K}$ or $T_{+}=122 \mathrm{~K}$ with longitudinal velocity $u_{+}=1 \mathrm{~cm} / \mathrm{sec}$. Taking into account the governing equation for $u$ and the boundary conditions $u_{ \pm}=1 \mathrm{~cm} / \mathrm{sec}$, the longitudinal velocity is found to be constant $u=1 \mathrm{~cm} / \mathrm{sec}$. The domain in the transformed coordinate $\zeta$ is typically given by $[-0.28,0.28]$.

For the temperature $T_{ \pm}=160 \mathrm{~K}$ at both ends the fluid stays away from the chemical stability limit whereas with the colder temperature $T_{ \pm}=122 \mathrm{~K}$ the fluid comes close to the chemical thermodynamic stability limit. This can be seen on Figure 1 where the thermodynamic coefficients $\gamma$ in the mixing layer is presented. For the larger temperature $T_{ \pm}=160 \mathrm{~K}$ the coefficient $\gamma$ significantly deviates from unity but still remains away from zero with a minimum value around $\gamma \approx 0.714$, whereas for $T_{ \pm}=122 \mathrm{~K}$ the coefficient $\gamma$ comes very close to the chemical thermodynamic stability limit with a minimum value around $\gamma \approx 3.510^{-3}$.

In Figure 2 are presented the hydrogen mole fraction $\mathrm{x}_{\mathrm{H}_{2}}(\zeta)$ as a function of $\zeta$ for both the ideal 
$(\gamma=1)$ and nonideal $\left(\gamma=-m_{1} m_{2} \mathcal{G}_{12} / m R T\right)$ diffusion models and in the two situations $T_{ \pm}=160 \mathrm{~K}$ and $T_{ \pm}=122 \mathrm{~K}$. When $T_{ \pm}=160 \mathrm{~K}$, the ideal and nonideal diffusion mole fraction profiles are essentially similar. On the contrary, when $T_{ \pm}=122 \mathrm{~K}$, it can be seen that the impact of nonidealities is dramatic. The nonideal diffusion mole fraction profile is much stiffer than the ideal diffusion one near chemical thermodynamic stability limit where the thermodynamic factor $\gamma$ vanishes. The sharp gradient of the mole fraction is due to the local quasi immiscibility of the two fluids. The limiting structure is then that of a contact discontinuity or equivalently that of a nonmixing layer [8]. An important consequence is the considerable decrease of mixing processes in such systems and the ideal diffusion model with $\gamma=1$ is unable to properly describe the relevant physics of diffusion.

The trajectories in the phase space $\left(\mathrm{x}_{\mathrm{H}_{2}}, T\right)$ corresponding to both boundary temperatures $T_{ \pm}=$ $160 \mathrm{~K}$ and $T_{ \pm}=122 \mathrm{~K}$ are further presented in Figure 3. In this phase diagram, the isocontours $\gamma=0.00, \gamma=0.25, \gamma=0.50$, and $\gamma=0.75$, of the thermodynamic parameter $\gamma$ are also presented. The forbidden zone $\gamma<0$, where the binary mixture is thermodynamically unstable, is also presented in grey color. It has already been observed that only nonideal diffusion fluxes properly represents the physics of diffusion near thermodynamic stability. However, it is also observed on Figure 3 that, with ideal diffusion fluxes, the forbidden unstable zone is crossed without even noticing it when $T_{ \pm}=122 \mathrm{~K}$. In particular, non-physical mixing states between the species are reached by this model. On the contrary, with the nonideal model, all trajectories remain in the physically admissible stable zone.

\section{Freely propagating premixed flames}

The impact of high pressure multicomponent transport models on the structure of freely propagating cryogenic supercritical flames is investigated [5].

\subsection{Planar flame equations}

We investigate freely propagating planar flames and denote by $x$ the coordinate normal to the combustion wave where $\boldsymbol{x}=(x, y, z)^{t}$. The velocity vector reads $\boldsymbol{v}=(u, 0,0)^{t}$ and the dependent unknowns have the structure

$$
\begin{aligned}
& T=T(t, x), \quad \rho=\rho(t, x), \quad \mathrm{y}_{i}=\mathrm{y}_{i}(t, x), \\
& u=u(t, x), \quad \tilde{p}=\widetilde{p}(t, x) .
\end{aligned}
$$

In the low Mach number regime, the momentum equation uncouples and would only be needed in order to evaluate the pressure corrector $\widetilde{p}$. For a steady flame, the resulting conservation equations for species mass and energy are found in the form

$$
\begin{aligned}
& \mathrm{my}_{i}^{\prime}+\mathcal{J}_{i}^{\prime}=m_{i} \omega_{i}, \quad i \in \mathfrak{S}, \\
& \mathrm{m}^{\prime}+\mathcal{J}_{\mathrm{e}}^{\prime}=0,
\end{aligned}
$$

where the superscript ' denotes the derivation with respect to the flame normal coordinate $x$ and $\mathrm{m}=\rho u$ is the mass flow rate, $\mathcal{J}_{i}$ the normal diffusion flux of the $i$ th species, and $\mathcal{J}_{\text {e }}$ the normal heat flux. The diffusion flux of the $i$ th species $\mathcal{J}_{i}=\left(\mathcal{J}_{i}, 0,0\right)^{t}, i \in \mathfrak{S}$, and the heat flux $\mathcal{J}_{\mathrm{e}}=\left(\mathcal{J}_{\mathrm{e}}, 0,0\right)^{t}$ reduce to their normal components. The flow rate $\mathrm{m}$ is constant from the total mass conservation equation $(\rho u)^{\prime}=0$ and is a nonlinear eigenvalue of the problem.

The boundary conditions at the origin are naturally written in the form $[84,85]$

$$
\begin{aligned}
& \mathrm{m}\left(\mathrm{y}_{i}(0)-\mathrm{y}_{i}^{\mathrm{fr}}\right)+\mathcal{J}_{i}(0)=0, \quad i \in \mathfrak{S}, \\
& \mathrm{m}\left(h(0)-h^{\mathrm{fr}}\right)+\mathcal{J}_{\mathrm{e}}(0)=0,
\end{aligned}
$$

where the superscript ${ }^{\text {fr }}$ refers to the fresh mixture. The downstream boundary conditions are in the form

$$
\mathrm{y}_{i}^{\prime}(+\infty)=0, \quad i \in \mathfrak{S}, \quad T^{\prime}(+\infty)=0,
$$

and the translational invariance of the model is removed by imposing a given temperature $T^{\mathrm{fx}}$ at a given arbitrary point $x^{\mathrm{fx}}$ of $[0, \infty)$

$$
T\left(x^{\mathrm{fx}}\right)-T^{\mathrm{fx}}=0,
$$




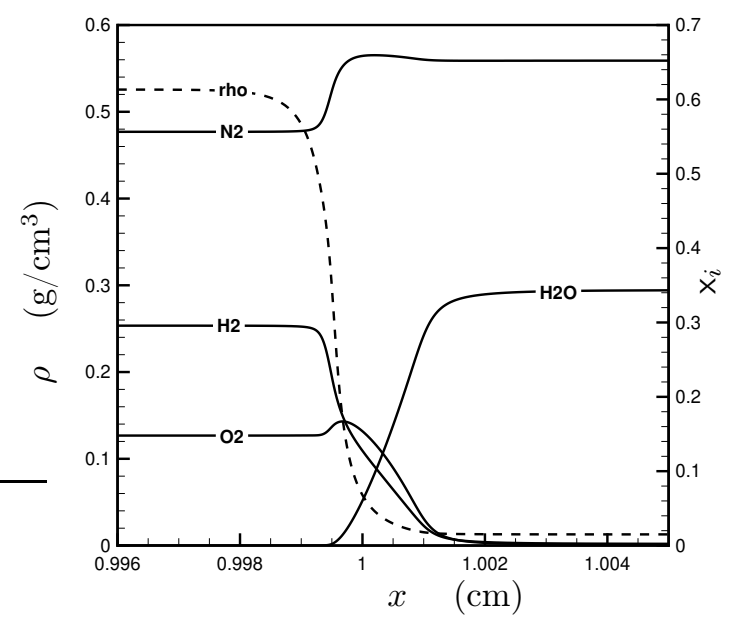

Figure 4: Mass density $\rho$ and mole fractions of major species in a freely propagating $\mathrm{H}_{2}$-Air stoichiometric flame structure at $\bar{p}=100 \mathrm{~atm}$ and $T^{\mathrm{fr}}=100 \mathrm{~K}$.

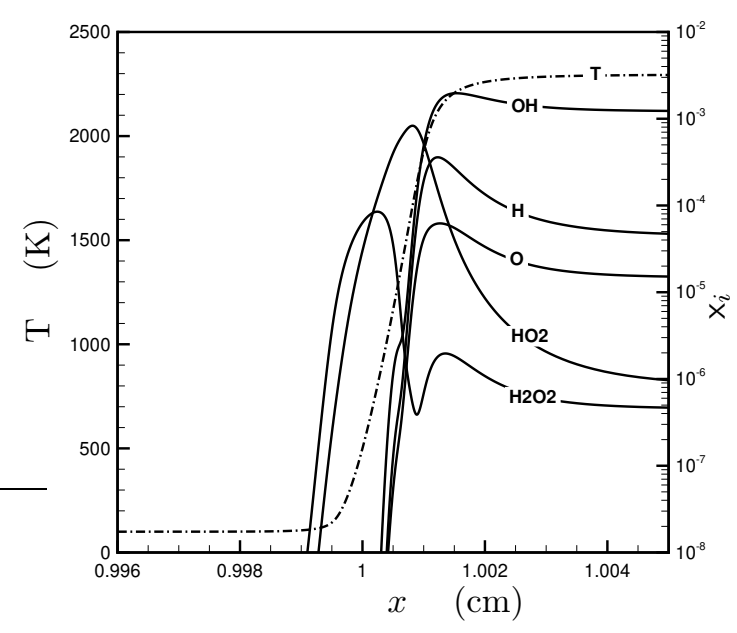

Figure 5: Temperature $T$ and mole fractions of minor species freely propagating $\mathrm{H}_{2}$-Air stoichiometric flame structure at $\bar{p}=100 \mathrm{~atm}$ and $T^{\mathrm{fr}}=100 \mathrm{~K}$.

where $T$ denotes the absolute temperature. These boundary and internal conditions (44)-(47) have first been used by Sermange [84] for $x^{\mathrm{fx}} \neq 0$ whereas $x^{\mathrm{fx}}=0$ was first chosen in reference [85]. The relations expressing the thermodynamic properties and chemical production rates have been discussed in Section 2 as well as the transport fluxes $\mathcal{J}_{i}, i \in \mathfrak{S}$, and $\mathcal{J}_{\mathrm{e}}$.

\subsection{A stoichiometric $\mathrm{H}_{2}$-Air flame}

We first discuss the structure of a typical premixed supercritical hydrogen/air flame. The reaction mechanism for hydrogen combustion is that developped by Warnatz [86, 87, 88]. Such complex chemistry flames depend on many kinetic parameters and a sensitivity analysis is notably presented in the book of Warnatz, Mass and Dibble [88]. The impact of nonidealities in multicomponent transport models - usually not taken into account - is performed in the next section. All flames are anchored by the condition $T\left(x^{\mathrm{fx}}\right)=T^{\mathrm{fx}}$ at $x^{\mathrm{fx}}=1 \mathrm{~cm}$ with $T^{\mathrm{fx}}=500 \mathrm{~K}$. To the authors' knowledge, there are no available experimental data on supercritical plane flames.

We consider a $\mathrm{H}_{2}$-Air flame with $\phi=1$ and $T^{\text {fr }}=100 \mathrm{~K}$ where $\phi$ denotes the equivalence ratio. Figure 4 presents the mass density $\rho$ and the major species mole fractions $\mathrm{x}_{i}, i \in \mathfrak{S}$, as function of the flame normal coordinate $x$ and Figure 5 presents the temperature $T$ and the minor species mole fractions. This flame is transcritical with respect to temperature - and remains supercritical with 


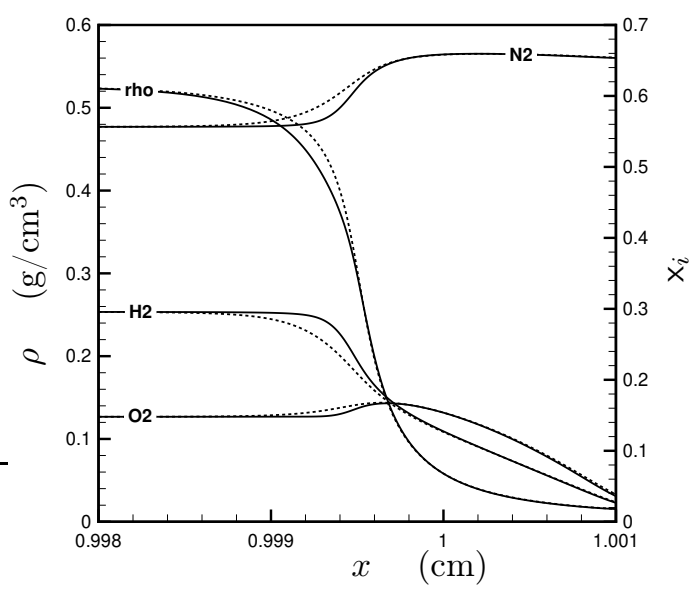

Figure 6: Impact of transport nonidealities in a freely propagating $\mathrm{H}_{2}$-Air stoichiometric flame at $\bar{p}=100 \mathrm{~atm}$ and $T^{\mathrm{fr}}=100 \mathrm{~K} ;$ — nonideal diffusion; $\mathbf{-}$ - ideal diffusion.

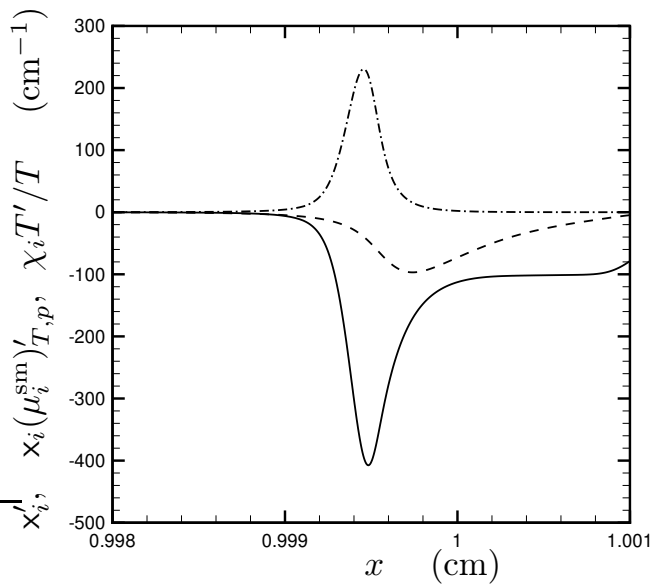

Figure 7: Hydrogen diffusion driving forces in a freely propagating $\mathrm{H}_{2}$-Air stoichiometric flame at $\bar{p}=100 \mathrm{~atm}$ and $T^{\mathrm{fr}}=100 \mathrm{~K} ;-\mathrm{x}_{i}^{\prime} ;--\mathrm{x}_{i}\left(\mu_{i}^{\mathrm{sm}}\right)_{T, p}^{\prime} ;-\chi_{i} T^{\prime} / T$.

respect to pressure - so that so that the density $\rho$ ranges from liquid-like values in the cold zone to gas-like values in the hot zone. Since the pressure is of $\bar{p}=100 \mathrm{~atm}$, the resulting flame front is much thinner than for atmospheric pressure flames. The flame front is roughly $40 \mu \mathrm{m}$ wide and presents large temperature and density gradients due to the low fresh gas temperature $T^{\mathrm{fr}}=100 \mathrm{~K}$ and to the combustion heat release. The mass flow rate is found to be $\mathrm{m}=0.981 \mathrm{~g} \mathrm{~cm}^{-2} \mathrm{~s}^{-1}$ and the flame speed of $u_{\mathrm{ad}}=1.866 \mathrm{~cm} \mathrm{~s}^{-1}$.

In $\mathrm{H}_{2}$-Air flames, the hydroperoxyl radical $\mathrm{HO}_{2}$ is generally formed early in the flame front by the three body reaction $\mathrm{H}+\mathrm{O}_{2}+\mathrm{M} \longrightarrow \mathrm{HO}_{2}+\mathrm{M}$ until it is dominated by the bimolecular reaction $\mathrm{H}+\mathrm{O}_{2} \longrightarrow \mathrm{OH}+\mathrm{O}$ at sufficiently high temperatures. The $\mathrm{HO}_{2}$ radical is then rapidly consumed through its reactions with the active radicals $\mathrm{H}$ and $\mathrm{OH}$. In high pressure flames, however, the reaction $\mathrm{H}+\mathrm{O}_{2}+\mathrm{M} \longrightarrow \mathrm{HO}_{2}+\mathrm{M}$ dominates $\mathrm{H}+\mathrm{O}_{2} \longrightarrow \mathrm{OH}+\mathrm{O}$ over a larger temperature domain since the crossing temperature is around $1400 \mathrm{~K}$ at atmospheric pressure but around $2100 \mathrm{~K}$ at $\bar{p}=100 \mathrm{~atm}$. As a result, high pressure $\mathrm{H}_{2}$-Air flames exhibit high concentrations of the $\mathrm{HO}_{2}$ radical and large concentrations of the $\mathrm{H}_{2} \mathrm{O}_{2}$ radical are subsequently obtained mainly through the reactions $\mathrm{HO}_{2}+$ $\mathrm{HO}_{2} \longrightarrow \mathrm{H}_{2} \mathrm{O}_{2}+\mathrm{O}_{2}$ and $\mathrm{H}_{2} \mathrm{O}+\mathrm{HO}_{2} \longrightarrow \mathrm{H}_{2} \mathrm{O}_{2}+\mathrm{OH}$. 


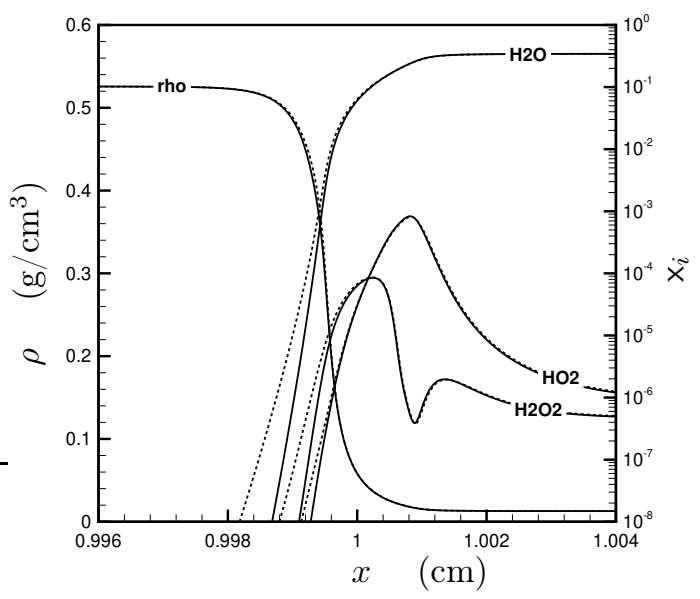

Figure 8: Impact of high pressure correctors of binary diffusion coefficients in a freely propagating $\mathrm{H}_{2}$ Air stoichiometric flame at $\bar{p}=100 \mathrm{~atm}$ and $T^{\mathrm{fr}}=100 \mathrm{~K} ;$ — nonideal diffusion; _ _ ideal diffusion.

\subsection{Impact of transport models}

The influence of transport nonidealities is investigated and we denote by $d_{j}$ the driving force of the $j$ th species in the normal direction $\boldsymbol{d}_{j}=\left(d_{j}, 0,0\right)^{t}$. We first compare the flame structure of Section 4.2 obtained with $d_{j}=\mathrm{x}_{j}\left(\mu_{j}\right)_{T, p}^{\prime}$ with the flame structure computed with $d_{j}=\mathrm{x}_{j}^{\prime}$ as for ideal diffusion. The corresponding density $\rho$ and mole fractions of major species $x_{i}, i \in \mathfrak{S}$, profiles are presented in Figure 6 as function of the flame normal coordinate $x$. We can see at once on Figure 6 that the full transport model prevents the migration of light cold $\mathrm{H}_{2}$ toward the flame front. The same behavior is also observed for the heavier molecules $\mathrm{O}_{2}$ and $\mathrm{N}_{2}$ and this is a main effect of nonidealities in premixed flames. This hindering of mass diffusion in the dense part of the flame leads to steeper gradients for hydrogen in the flame front and the mixture loses this impermeability only by warming.

To gain more insight into diffusive processes, we present in Figure 7 the contributions to the overall diffusion force $d_{j}$ of the ideal part $\mathrm{x}_{j}^{\prime}$ and of the nonideal part $\mathrm{x}_{j}\left(\mu_{j}^{\mathrm{sm}}\right)_{T}^{\prime}$ for the $\mathrm{H}_{2}$ species as well as the Soret term $\chi_{j}(\log T)^{\prime}$. The nonideal contribution $\mathrm{x}_{j}\left(\mu_{j}^{\mathrm{sm}}\right)_{T}^{\prime}$ is strongly bound to density gradients and is negligible out of a narrow domain located early in the flame front. Neither the mole fraction gradient $\mathrm{x}_{j}^{\prime}$ nor the Soret term $\chi_{j}(\log T)^{\prime}$ are confined in this high density region as can be seen in Figure 7 . The main effect of the nonideal parts of the diffusion forces is mainly to hinder mass diffusion between the denser liquid-like region and the flame front. The impact of transport nonidealities may therefore become stronger when temperature and density profiles stiffen.

We finally compare in Figure 8, the flame structure obtained with the dilute gas binary diffusion coefficients $\mathcal{D}_{i j}^{\text {PG }}$ with the flame structure of Section 4.2 computed with the high pressure binary diffusion coefficients $\mathcal{D}_{i j}=\mathcal{D}_{i j}^{\mathrm{PG}} / \Upsilon_{i j}$. The density $\rho$ and the mole fractions $x_{i}, i \in \mathfrak{S}$, of some radical species profiles are presented as function of the flame normal coordinate $x$. It can be seen that for the species $\mathrm{H}_{2} \mathrm{O}, \mathrm{HO}_{2}$, and $\mathrm{H}_{2} \mathrm{O}_{2}$ the influence of such high pressure corrections to binary diffusion coefficient takes place in a narrow zone between the dense fluid region and the flame front. The other species are mostly absent from this zone and are essentially not modified.

\section{Strained flames}

The impact of transport nonidealities on the structure of a high pressure strained diffusion flame is investigated [6].

\subsection{Strained flame equations}

Self-similar solutions of multicomponent fluid equations in the small Mach number limit notably include stagnation point flows. Such strained flows are of fundamental importance for understanding the effect of strain due to turbulence on flame fronts $[3,4,6]$. The spatial coordinates are denoted by $\boldsymbol{x}=(x, y, z)^{t}$ where $x$ is the transverse coordinate, $y$ the flame normal coordinate, and the components of the velocity 
vector are denoted by $\boldsymbol{v}=(u, v, 0)^{t}$. The Mach number is assumed to be small and the pressure is decomposed in the form $p=\bar{p}+\widetilde{p}$ where $\bar{p}$ is the ambient pressure constant in space and $\widetilde{p}$ the fluid dynamic perturbation with the ratio $\tilde{p} / \bar{p}$ of the order of the square of the Mach number. The solution is assumed to have the following self-similar structure [52]

$$
\begin{aligned}
& T=T(t, y), \quad \rho=\rho(t, y), \quad \mathrm{y}_{i}=\mathrm{y}_{i}(t, y), \\
& u=x \widetilde{u}(t, y), \quad \quad v=v(t, y), \quad \widetilde{p}=-\frac{1}{2} J x^{2}+\widehat{p}(t, y),
\end{aligned}
$$

where $J$ is a reduced pressure gradient. It is then obtained from the expression of multicomponent transport fluxes that they are in the normal direction $\mathcal{J}_{i}=\left(0, \mathcal{J}_{i}, 0\right)^{t}, i \in \mathfrak{S}$, and $\mathcal{J}_{\mathrm{e}}=\left(0, \mathcal{J}_{\mathrm{e}}, 0\right)^{t}$ where $\mathcal{J}_{i}(t, y), i \in \mathfrak{S}$, and $\mathcal{J}_{\mathrm{e}}(t, y)$ denote the normal flux components. Substituting these expressions in the governing equations in the small Mach number limit, the normal momentum equation is found to uncouple from the others whereas the tangential momentum equation has all its terms proportional to $x$. The resulting steady equations then form a boundary value problem only involving the normal coordinate $y$

$$
\begin{aligned}
\rho \widetilde{u}+\partial_{y}(\rho v) & =0, \\
\rho v \partial_{y} \mathrm{y}_{i}+\partial_{y} \mathcal{J}_{i} & =m_{i} \omega_{i}, \quad i \in \mathfrak{S}, \\
\rho \widetilde{u}^{2}+\rho v \partial_{y} \widetilde{u}-J+\partial_{y}\left(\eta \partial_{y} \widetilde{u}\right) & =0, \\
\rho v \partial_{y} h+\partial_{y} \mathcal{J}_{\mathrm{e}} & =0 .
\end{aligned}
$$

Such equations are also valid for axisymmetric flows with a factor 2 in front of $\rho \widetilde{u}$ in (48). The relations expressing the thermodynamic properties and chemical production rates have been discussed in Section 2 as well as the transport fluxes $\mathcal{J}_{i}, i \in \mathfrak{S}$, and $\mathcal{J}_{\mathrm{e}}$.

The boundary conditions are typically in the form

$$
\begin{aligned}
T(-\infty) & =T_{-}, & T(+\infty) & =T_{+}, \\
\mathrm{y}_{i}(-\infty) & =\mathrm{y}_{i-}, & \mathrm{y}_{i}(+\infty) & =\mathrm{y}_{i+}, \\
\widetilde{u}(-\infty) & =\alpha \sqrt{\rho_{+} / \rho_{-}}, & \widetilde{u}(+\infty) & =\alpha,
\end{aligned}
$$

where the superscript + is associated with the liquid like fluid coming from the positive side on the right, the superscript - to the gas like fluid coming from the negative side on the left. The imposed strain rate $\alpha$ is related to the pressure gradient with the relation

$$
\alpha=\left(J / \rho_{+}\right)^{1 / 2},
$$

and the stagnation plane is located for convenience at the origin with

$$
v(0)=0 .
$$

\subsection{A strained $\mathrm{H}_{2}-\mathrm{O}_{2}$ diffusion flame}

We investigate a $\mathrm{H}_{2}-\mathrm{O}_{2}$ strained diffusion flame and the reaction mechanism of O'Conaire et al. [89] has been used in the calculations. A detailed sensitivity analysis with respect to reaction constants as well as binary diffusion coefficients for strained flames has been presented by Holley at al. [90]. In the oxygen stream we have $T_{+}=100 \mathrm{~K}$ and $\mathrm{y}_{\mathrm{O}_{2}+}=1$, in the hydrogen stream $T_{-}=300 \mathrm{~K}$ and $\mathrm{y}_{\mathrm{H}_{2-}}=1$, the ambient pressure is $\bar{p}=100$ atm and the strain rate is $\alpha=10000 \mathrm{~s}^{-1}$. Figure 9 presents the density and the major species mole fractions as function of the normal coordinate $y$ whereas Figure 10 presents the temperature and the minor species mole fractions. An important feature of this flame structure is the large densities encountered in the oxygen injection for positive $y$. This density is indeed as high as $1.12 \mathrm{~g} \cdot \mathrm{cm}^{-3}$ close to the density of liquid oxygen and very rapidly decreases to gas-like values for decreasing $y$. These large variations are due to the fact that the flame is supercritical with respect to pressure but transcritical with respect to temperature.

The whole flame structure is around $50 \mu \mathrm{m}$ wide and on the $\mathrm{O}_{2}$ side, there is an early formation of the metastable $\mathrm{H}_{2} \mathrm{O}_{2}$ radical through reactions first forming $\mathrm{HO}_{2}$. Due to the low diffusivity of light radicals in the liquid-like oxygen stream, there is also an important shift of light radicals toward the hydrogen side, especially atomic hydrogen. The temperature profile is bell shaped and the maximal temperature $T^{\max }=3747 \mathrm{~K}$ is reached in the vicinity of $y=19.6 \mu \mathrm{m}$, the crossing position of the fuel and oxydant mole fraction where $\mathrm{x}_{\mathrm{H}_{2}}=\mathrm{x}_{\mathrm{O}_{2}}=0.078$. 


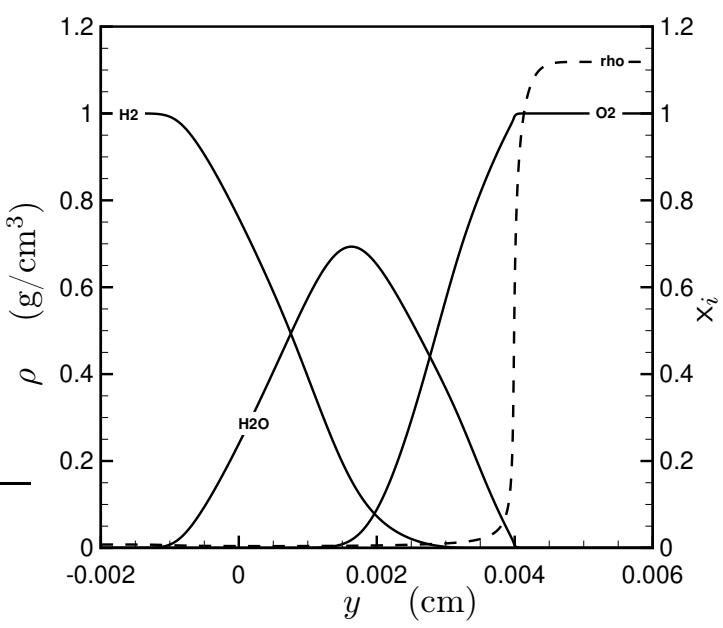

Figure 9: Mass density $\rho$ and mole fractions of major species in a counterflow $\mathrm{H}_{2}-\mathrm{O}_{2}$ diffusion flame, with $T_{+}=100 \mathrm{~K}, \mathrm{y}_{2^{2}}=1, T_{-}=300 \mathrm{~K}, \mathrm{y}_{\mathrm{H}^{-}}=1, \alpha=10000 \mathrm{~s}^{-1}$, and $\bar{p}=100 \mathrm{~atm}$.

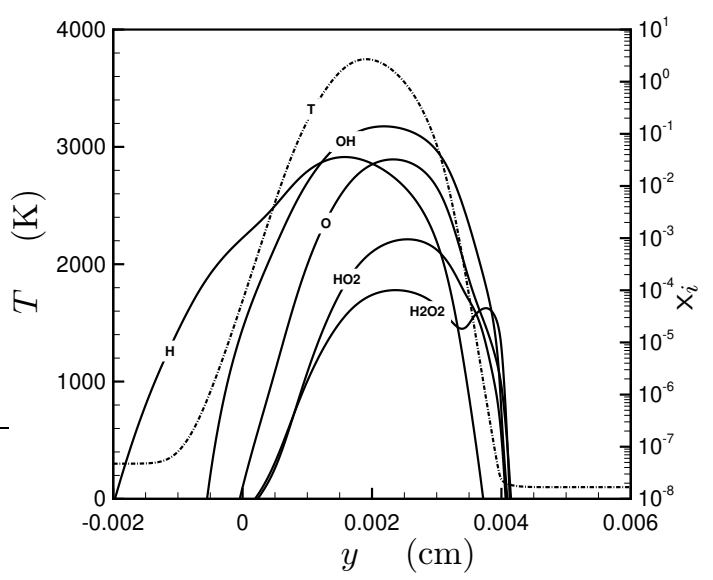

Figure 10: Temperature $T$ and mole fraction of minor species in a counterflow $\mathrm{H}_{2}-\mathrm{O}_{2}$ diffusion flame, with $T_{+}=100 \mathrm{~K}, \mathrm{y}_{\mathrm{O}_{2+}}=1, T_{-}=300 \mathrm{~K}, \mathrm{y}_{\mathrm{H}_{2-}}=1, \alpha=10000 \mathrm{~s}^{-1}$, and $\bar{p}=100 \mathrm{~atm}$.

\subsection{Impact of nonideal transport}

We present in Figure 11 the ideal diffusion driving force $\partial_{y} \mathrm{x}_{\mathrm{O}_{2}}$ of the $\mathrm{O}_{2}$ species as well as the full diffusion driving force $d_{\mathrm{O}_{2}}$ that includes the nonideal part and the Soret contribution as function of the normal coordinate $y$ and the same quantities are also presented for the $\mathrm{H}_{2} \mathrm{O}$ species. The nonideal part of the diffusion force $\mathrm{x}_{\mathrm{O}_{2}}\left(\partial_{y} \mu_{\mathrm{O}_{2}}^{\mathrm{sm}}\right)_{T}$ plays here an anti-diffusion effect in a very narrow sheet around the high density gradient zone. Its influence is then to prevent diffusion of $\mathrm{O}_{2}$ molecules from the dense core to the flame. This hindering of diffusion in condensed like regions has already been observed in the premixed planar situation.

The influence of nonidealities is thus mainly to reduce the diffusivity of $\mathrm{H}_{2} \mathrm{O}$ inside the dense $\mathrm{O}_{2}$ core and to prevent that of oxygen in the mixture. On the contrary, with the ideal model, diffusion is artificially increased and the oxygen mole fraction is smoothed. This smoothing can be shown in Figure 12 where the cold zone has been enlarged. The oxygen mole fraction profile then presents a kind of bump in this steep density gradient region, bump that can be obliterated by neglecting the nonideal diffusion. Taking into account the thermal diffusion does not modify this conclusion as its own influence is mainly located inside the flame and not in the pseudo-interface region.

Therefore, for such a strain diffusion flame with pure oxygen on the cold side, using the ideal diffusion driving forces does not essentially change the flame structure and the radicals profile. The nonideal transport influence on the $\mathrm{O}_{2}$ pseudo-vaporizing mass transfer rate is weak compared to thermal effects. The multi-species flame sheet is separated from the pure species dense $\mathrm{O}_{2}$ core by this 


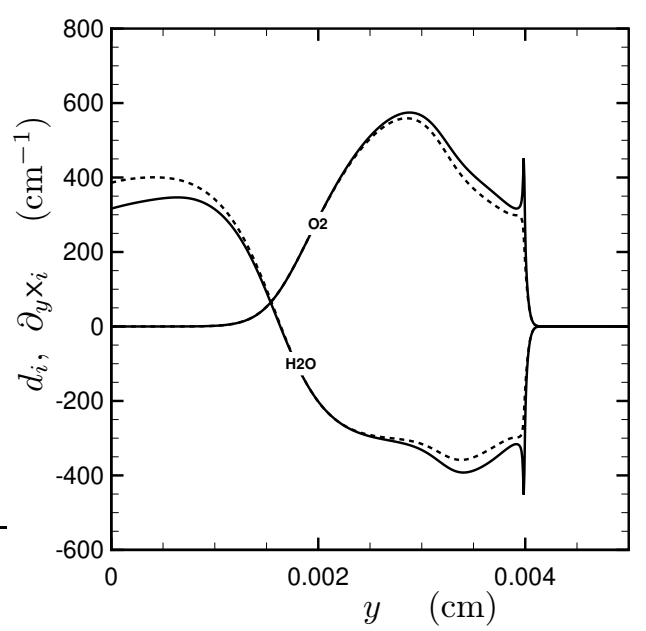

Figure 11: Oxygen and water diffusion driving forces in a in a counterflow $\mathrm{H}_{2}-\mathrm{O}_{2}$ diffusion flame, with $T_{+}=100 \mathrm{~K}, \mathrm{y}_{\mathrm{O}_{2+}}=1, T_{-}=300 \mathrm{~K}, \mathrm{y}_{\mathrm{H}_{2-}}=1, \alpha=10000 \mathrm{~s}^{-1}, \bar{p}=100 \mathrm{~atm} ; \boldsymbol{-}-d_{i} ;$ - $\partial_{y} \mathrm{x}_{i}$.

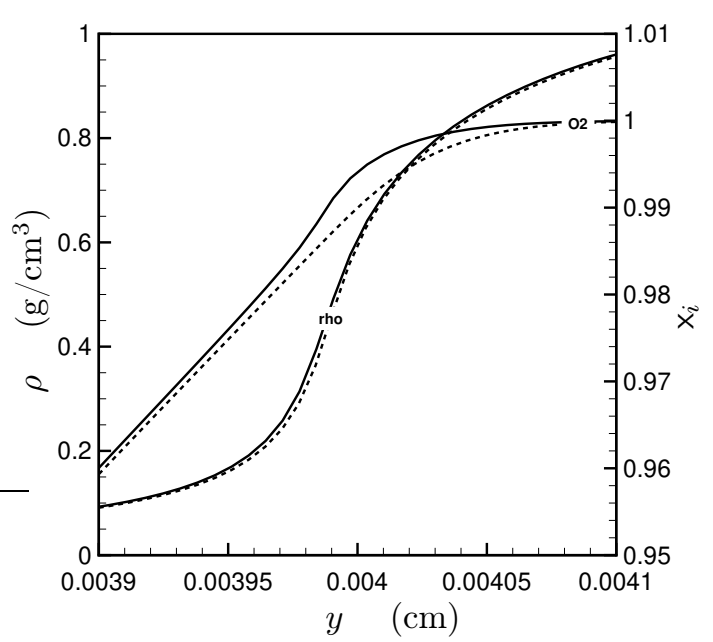

Figure 12: Density $\rho$ and oxygen mole fraction in a counterflow $\mathrm{H}_{2}-\mathrm{O}_{2}$ diffusion flame, with $T_{+}=100 \mathrm{~K}$, $\mathrm{y}_{\mathrm{O}_{2}+}=1, T_{-}=300 \mathrm{~K}, \mathrm{y}_{\mathrm{H}_{2-}}=1, \alpha=10000 \mathrm{~s}^{-1}, \bar{p}=100 \mathrm{~atm} ;$ — nonideal diffusion; _ - ideal diffusion.

thermally controlled pseudo-vaporizing front, justifying subgrid models based on pseudo-vaporizing interface consideration [91].

\section{Transcritical diffusion flames}

We investigate multicomponent transport fluxes in a nonideal dense reactive fluid model with diffuse interfaces of Van der Waals/Korteweg/Cahn-Hilliard type [92, 93, 94, 95, 96, 97, 98, 99, 100, 101, 102, 103, 79]. The model is valid at all pressures and may be used to investigate the ignition phase in rocket engines as well as the combustion of liquid droplets [104, 105, 106, 107, 108, 110, 9, 111]. Such a model may be used in particular to investigate flames that are transcritical with respect to pressure [7].

\subsection{A Van der Waals/Korteweg/Cahn-Hilliard fluid}

Diffuse interface models describe the continuous change of fluid properties across liquid/gas interfaces in subcritical conditions. Such diffuse interface models may be derived from the second gradient theory of Van der Waals, Korteweg, Cahn and Hilliard [92, 93, 94, 95, 96, 97, 98, 99, 100, 101, 102, 103, 104, $105,106,107,108,110,79]$. Statistical mechanics of systems that are highly inhomogeneous has also 
led to mean field theories of liquid/gas interfaces and to direct links between capillary phenomena and intermolecular forces as established by Rowlinson and Widom [97]. Second gradient diffuse interface models have been successfully used to describe near critical points [97], the dynamics of contact line [98] as well as complex liquid/gas interfaces with topological changes [99, 100, 101] and are compatible with the limiting free boundary problem when the interface thickness goes to zero [99].

The free energy per unit volume in the second gradient theory is typically in the form

$$
\mathcal{F}=\mathcal{F}^{0}+\sum_{i, j \in \mathfrak{S}} \kappa_{i j} \nabla \rho_{i} \cdot \nabla \rho_{j}
$$

where $\mathfrak{S}=\{1, \ldots, n\}$ denotes the species indexing set, $n$ the number of species, $\mathcal{F}^{0}$ the bulk free energy, $\rho_{i}$ the partial density of the $i$ th species, $\nabla$ the usual differential operator and $\kappa_{i j}, i, j \in \mathfrak{S}$, the capillary coefficients. The superscript ${ }^{0}$ is used to denote standard bulk phase thermodynamic properties that do not involve gradients. The free energy $\mathcal{F}^{0}$ only depends on the partial densities $\rho_{1}, \ldots, \rho_{n}$ and the absolute temperature $T$ whereas the gradient squared term $\sum_{i, j \in \mathfrak{S}} \kappa_{i j} \nabla \rho_{i} \cdot \nabla \rho_{j}$ in $\mathcal{F}$ represents the excess free energy of the interfacial region. This gradient term may be interpreted from attractive long range molecular interactions and the capillary coefficients $\kappa_{i j}$ related to the pair correlation function [97]. From the expression of the free energy (57) and the classical thermodynamic relation $\mathbb{D} \mathcal{F}^{0}=-\mathcal{S}^{0} \mathbb{D} T+\sum_{i \in \mathfrak{S}} g_{i}^{0} \mathbb{D} \rho_{i}$, assuming that the capillarity coefficients $\kappa_{i j}, i, j \in \mathfrak{S}$, are constant for the sake of simplicity, the differential of the free energy is found in the form

$$
\mathbb{D} \mathcal{F}=-\mathcal{S}^{0} \mathbb{D} T+\sum_{i \in \mathfrak{S}} g_{i}^{0} \mathbb{D} \rho_{i}+\sum_{i \in \mathfrak{S}} \boldsymbol{\phi}_{i} \cdot \mathbb{D} \nabla \rho_{i},
$$

where $\mathcal{S}^{0}$ denotes the bulk entropy per unit volume, $g_{i}^{0}$ the bulk Gibbs function of the $i$ th species per unit mass, and the vectors $\phi_{i}, i \in \mathfrak{S}$, are given by

$$
\phi_{i}=\sum_{j \in \mathfrak{S}}\left(\kappa_{i j}+\kappa_{j i}\right) \nabla \rho_{j}, \quad i \in \mathfrak{S} .
$$

Using the thermodynamic relations $\partial_{T} \mathcal{F}=-\mathcal{S}$ and $\partial_{\rho_{i}} \mathcal{F}=g_{i}, i \in \mathfrak{S}$, where $\mathcal{S}$ denotes the entropy per unit volume and $g_{i}$ the Gibbs function per unit mass of the $i$ th species, the identity (58) implies that $\mathcal{S}=\mathcal{S}^{0}$ and $g_{i}=g_{i}^{0}$. The Gibbs function and enthalpy per unit volume are thus given by their standard value $\mathcal{G}=\mathcal{G}^{0}$ and $\mathcal{H}=\mathcal{H}^{0}$ whereas the energy per unit volume is given by $\mathcal{E}=\mathcal{E}^{0}+\sum_{i, j \in \mathfrak{S}} \kappa_{i j} \nabla \rho_{i} \cdot \nabla \rho_{j}$ and the pressure by $p=p^{0}-\sum_{i, j \in \mathfrak{S}} \kappa_{i j} \nabla \rho_{i} \cdot \nabla \rho_{j}$.

When the interface thickness goes to zero, the diffuse interface model yields the traditional limiting free boundary problem with surface tension [99]. In particular, surface tension - that is meaningful only at the macroscopic limit - may be derived from the capillary coefficients and the state law [79]. These relations may be used in practice in order to obtain physical values for the capillary coefficients $\kappa_{i j}, i, i \in \mathfrak{S}$, that are generally temperature dependent, by using the state law and fitting surface tension experimental data [103]. Such coefficients are typically in the form of an exponential of a polynomial in $\log \left(1-T / T^{\mathrm{c}}\right)$, where $T^{\mathrm{c}}$ is a critical temperature and are thus only defined in the subcritical domain [103]. The capillary coefficient $\kappa$ for oxygen at is of the order of $\kappa=2.510^{-7} \mathrm{~g}^{-1} \mathrm{~cm}^{7} \mathrm{~s}^{-2}$ at temperature $100 \mathrm{~K}[103,109]$ and the resulting diffuse interfaces are then very thin, typically of the order of a few nanometers $[97,108,107]$. The diffuse interface model allows to faithfully reproduce the structure of such interfaces using numerical grids with nanometer characteristic lengths [108, 107].

Although such detailed simulations can be obtained with the diffuse interface model, we want to obtain in this paper computationally feasible numerical flame models taking into account the limitation on grid resolution. In this situation, one is forced to use larger values of the capillary coefficients leading to artificially thickened interfaces and to larger values of surface tension. This implicitly assumes that there is a scale separation so that interface thickening and the corresponding larger numerical surface tension do not significantly modify the flame under investigation. In addition, our aim is to extend the diffuse interface model to supercritical conditions in order to encompass all pressures. We thus have to extend the domain of definition of capillary coefficients with finite values for all pressures and, in practice, we have used constant values for capillary parameters. In the subcritical domain, the resulting diffuse interface model then allows the computation of a continuous liquid/gas interface whereas in the supercritical domain the model may ease the numerical solution of sharp pseudo vaporizing transition layers. The Cahn-Hilliard derivation of the extra gradient energy term (57) as well as its link with attractive long range molecular interactions further indicates that there is also a capillary coefficients $\kappa$ in the supercritical domain. 
The governing equation in a Van der Waals/Korteweg fluid associated with the free energy (57) are in the form

$$
\begin{array}{r}
\partial_{t} \rho_{i}+\nabla \cdot\left(\rho_{i} \boldsymbol{v}\right)+\boldsymbol{\nabla} \cdot \mathcal{J}_{i}=m_{i} \omega_{i}, \quad i \in \mathfrak{S}, \\
\partial_{t}(\rho \boldsymbol{v})+\nabla \cdot(\rho \boldsymbol{v} \otimes \boldsymbol{v})+\nabla \cdot \mathcal{P}=0, \\
\partial_{t}\left(\rho\left(e+\frac{1}{2}|\boldsymbol{v}|^{2}\right)\right)+\boldsymbol{\nabla} \cdot\left(\rho \boldsymbol{v}\left(e+\frac{1}{2}|\boldsymbol{v}|^{2}\right)\right)+\nabla \cdot \mathcal{Q}=0,
\end{array}
$$

where $\mathcal{P}$ denotes the pressure tensor and $\mathcal{Q}$ denotes the energy flux using similar notation as for ordinary fluids. In order to derive proper expressions for the diffusion fluxes $\mathcal{J}_{i}, i \in \mathfrak{S}$, the pressure tensor $\mathcal{P}$ and the energy flux $\mathcal{Q}$, one may then use a method similar to that of the thermodynamics of irreversible processes $[26,99,101,79]$. Using the general form of the free energy, it is found that the pressure tensor and the total heat flux are in the form

$$
\begin{aligned}
& \mathcal{P}=p \boldsymbol{I}+\sum_{i \in \mathfrak{S}}\left(\nabla \rho_{i} \otimes \boldsymbol{\phi}_{i}-\rho_{i} \boldsymbol{\nabla} \cdot \boldsymbol{\phi}_{i} \boldsymbol{I}\right)+\boldsymbol{\Pi} \\
& \mathcal{Q}=-\sum_{i \in \mathfrak{S}} \boldsymbol{\phi}_{i}\left(m_{i} \omega_{i}-\boldsymbol{\nabla} \cdot \mathcal{J}_{i}-\rho_{i} \boldsymbol{\nabla} \cdot \boldsymbol{v}\right)+\mathcal{J}_{\mathrm{e}}+\mathcal{P} \cdot \boldsymbol{v}
\end{aligned}
$$

where $\boldsymbol{\Pi}$ is the viscous tensor and $\mathcal{J}_{\mathrm{e}}$ the dissipative heat flux. The dissipative transport fluxes $\boldsymbol{\Pi}$, $\mathcal{J}_{i}, i \in \mathfrak{S}$, and $\mathcal{J}_{\mathrm{e}}$ are also found in the form

$$
\begin{aligned}
& \boldsymbol{\Pi}=-\mathfrak{v} \boldsymbol{\nabla} \cdot \boldsymbol{v} \boldsymbol{I}-\eta\left(\boldsymbol{\nabla} \boldsymbol{v}+\boldsymbol{\nabla} \boldsymbol{v}^{t}-\frac{2}{3} \boldsymbol{\nabla} \cdot \boldsymbol{v} \boldsymbol{I}\right), \\
& \mathcal{J}_{i}=-\sum_{j \in \mathfrak{S}} L_{i j} \boldsymbol{\nabla}\left(\frac{g_{j}^{0}}{T}-\frac{\boldsymbol{\nabla} \cdot \boldsymbol{\phi}_{j}}{T}\right)-L_{i e} \boldsymbol{\nabla}\left(\frac{-1}{T}\right), \\
& \mathcal{J}_{\mathrm{e}}=-\sum_{i \in \mathfrak{S}} L_{e i} \boldsymbol{\nabla}\left(\frac{g_{i}^{0}}{T}-\frac{\boldsymbol{\nabla} \cdot \boldsymbol{\phi}_{i}}{T}\right)-L_{e e} \boldsymbol{\nabla}\left(\frac{-1}{T}\right),
\end{aligned}
$$

where $\mathfrak{v}$ denotes the volume viscosity, $\eta$ the shear viscosity, $L_{i j}, i, j \in \mathfrak{S} \cup\{e\}$, the mass and heat transport coefficients. The matrix of mass and heat transport coefficients $L$ defined by $L=\left(L_{i j}\right)_{i, j \in \mathfrak{S} \cup\{e\}}$ is symmetric positive semi-definite with nullspace spanned by the vector $(1, \ldots, 1,0)^{t}$ as for ordinary fluids [26]. The fluxes $\mathcal{J}_{i}, i \in \mathfrak{S}$, and $\mathcal{J}_{\text {e }}$ are obtained here in their thermodynamic form. In the pressure tensor $\mathcal{P}$, the component $\mathcal{P}-p^{0} \boldsymbol{I}-\boldsymbol{\Pi}$ represents the reversible part associated with interstitial work. In the diffusion fluxes, the extra terms $\boldsymbol{\nabla} \cdot \boldsymbol{\phi}_{j}, j \in \mathfrak{S}$, involving the vectors $\boldsymbol{\phi}_{i}$ notably lead to Cahn-Hilliard type equations.

\subsection{A simplified Van der Waals/Korteweg fluid}

We introduce in this section a simplified model obtained by assuming that all capillary coefficients are equal $\kappa_{i j}=\frac{1}{2} \kappa, i, j \in \mathfrak{S}$. This model is well justified when there are large density gradients at the interface and the interface composition is essentially frozen so that the total mass $\rho$ density may be used as an interface parameter [7]. In this situation the free energy is in the form

$$
\mathcal{F}=\mathcal{F}^{0}+\frac{1}{2} \kappa|\nabla \rho|^{2}
$$

where $\mathcal{F}^{0}$ denotes the bulk free energy and $\kappa$ the common value of the capillary coefficients, and we also have $\phi_{1}=\cdots=\phi_{n}=\kappa \nabla \rho$. The pressure $p$ and the energy per unit volume are then given $p=p^{0}-\kappa|\nabla \rho|^{2}$ and $\mathcal{E}=\mathcal{E}^{0}+\frac{1}{2} \kappa|\nabla \rho|^{2}$. Taking into account the simplified expression (68), as well as natural mass constraints, the resulting pressure tensor $\mathcal{P}$ and energy flux $\mathcal{Q}$ are found in the form

$$
\begin{aligned}
& \mathcal{P}=p \boldsymbol{I}+\kappa \nabla \rho \otimes \nabla \rho-\kappa \rho \Delta \rho \boldsymbol{I}+\boldsymbol{\Pi}, \\
& \mathcal{Q}=\kappa \rho \boldsymbol{\nabla} \cdot \boldsymbol{v} \nabla \rho+\mathcal{J}_{\mathrm{e}}+\mathcal{P} \cdot \boldsymbol{v},
\end{aligned}
$$

whereas the multicomponent fluxes $\mathcal{J}_{i}, i \in \mathfrak{S}$, and $\mathcal{J}_{\text {e }}$ are unchanged. Indeed the vector of extra gradient terms in the generalized diffusion driving forces

$$
\left(\nabla\left(\frac{\nabla \cdot \phi_{1}}{T}\right), \ldots, \nabla\left(\frac{\nabla \cdot \phi_{n}}{T}\right), 0\right)^{t}=\frac{\nabla \cdot(\kappa \nabla \rho)}{T} \mathcal{U}
$$


is in then in the nullspace $N(L)=\mathbb{R} \mathcal{U}$ of the matrix $L$ with $\mathcal{U}=(1, \ldots, 1,0)^{t}$. In the pressure tensor $\mathcal{P}$ the new contributions are $-\kappa \rho \Delta \rho \boldsymbol{I}$, the Korteweg tensor $\kappa \nabla \rho \otimes \nabla \rho$ and the pressure $p$ also differs from $p^{0}$. These extra terms associated with capillary phenomena do not produce entropy and are associated with interstitial work which is a reversible process. The heat flux $\mathcal{Q}-\mathcal{P} \cdot \boldsymbol{v}$ also contain a supplementary term $\kappa \rho \boldsymbol{\nabla} \cdot \boldsymbol{v} \boldsymbol{\nabla} \rho$ induced by capillarity. When the capillary coefficient vanish, the standard fluid pressure tensor is recovered as well as the standard energy flux.

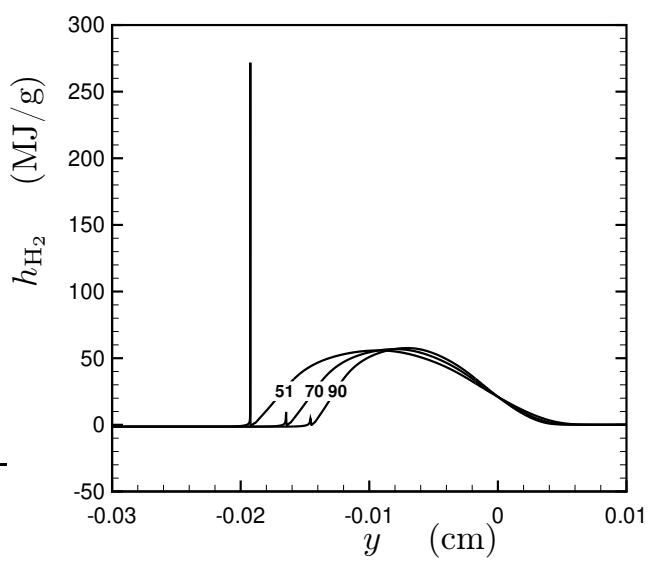

Figure 13: Hydrogen partial enthalpies in supercritical $\mathrm{H}_{2}-\mathrm{O}_{2}$ diffusion flames, with $T_{-}=100 \mathrm{~K}$, $\mathrm{y}_{\mathrm{O}_{2}-}=1, T_{+}=300 \mathrm{~K}, \mathrm{y}_{\mathrm{H}_{2}+}=1, \alpha=1000 \mathrm{~s}^{-1}$ and pressures $p^{\infty}=51,70,90 \mathrm{~atm}$.

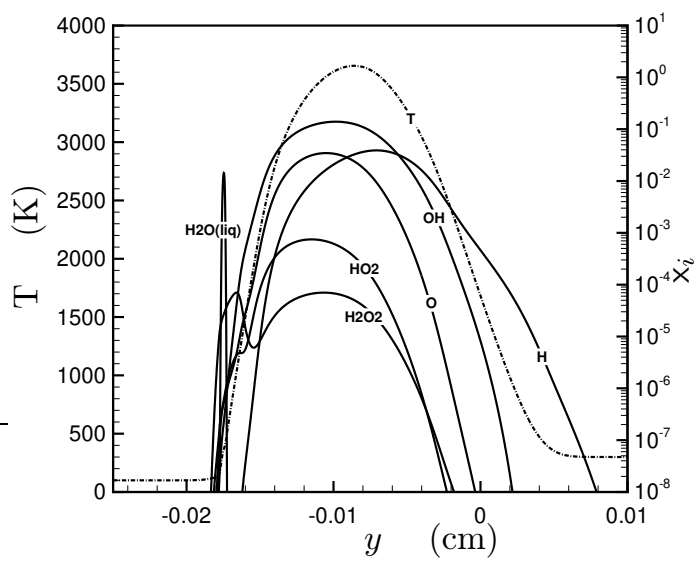

Figure 14: Temperature $T$ and species mole fractions in a diffuse interface $\mathrm{H}_{2}-\mathrm{O}_{2}$ diffusion flame at $p^{\infty}=60$ bars with $T_{-}=100 \mathrm{~K}, \mathrm{y}_{\mathrm{O}_{2}-}=1, T_{+}=300 \mathrm{~K}, \mathrm{y}_{\mathrm{H}_{2}+}=1, \alpha=1000 \mathrm{~s}^{-1}$

\subsection{Singularities in transport fluxes}

The dissipative fluxes $\mathcal{J}_{i}, i \in \mathfrak{S}$, and $\mathcal{J}_{\text {e }}$ are evaluated in their thermodynamic form. To this aim, it is necessary to evaluate the mass and heat transport coefficient matrix $L$ of size $n+1$ that can be written in the form $[5,55]$

$$
L=\left(\begin{array}{cc}
\mathcal{D} & \mathcal{D} \mathfrak{h} \\
(\mathcal{D} h)^{t} & \lambda T^{2}+\langle\mathcal{D} \mathfrak{h}, \hat{h}\rangle
\end{array}\right),
$$

where $\mathcal{D}=\left(\mathcal{D}_{i j}\right)_{i, j \in \mathfrak{S}}$ and $\hbar=\left(\hbar_{1}, \ldots, \hbar_{n}\right)^{t}$ are given by

$$
\mathcal{D}_{i j}=\rho \mathrm{y}_{i} \mathrm{y}_{j} \frac{m}{R} D_{i j}, \quad h_{i}=h_{i}+R T \frac{\widetilde{\chi}_{i}}{m_{i}}
$$




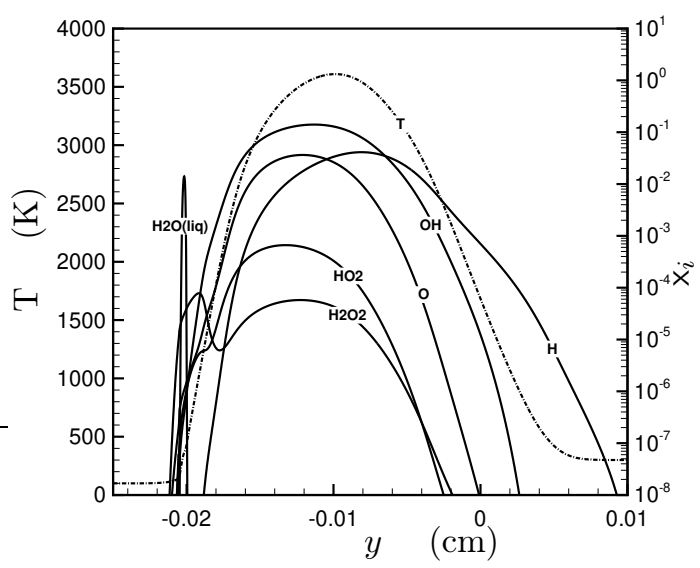

Figure 15: Temperature $T$ and species mole fractions in a diffuse interface $\mathrm{H}_{2}-\mathrm{O}_{2}$ diffusion flame at $p^{\infty}=45$ bars with $T_{-}=100 \mathrm{~K}, \mathrm{y}_{2^{-}}=1, T_{+}=300 \mathrm{~K}, \mathrm{y}_{\mathrm{H}_{2}}=1, \alpha=1000 \mathrm{~s}^{-1}$

For an all pressure flame model, however, some care must be taken, in order to evaluate the species specific enthalpies $h_{i}=\left(\partial_{\mathrm{y}_{i}} h\right)_{T, p^{0}, y_{l}}, i \in \mathfrak{S}$, appearing in (72) in the presence of mechanical critical points where $\left(\partial_{\rho} p^{0}\right)_{T, y_{l}}=0$. We indeed have the identity

$$
h_{k}=\left(\partial_{y_{k}} h\right)_{T, p^{0}, y_{l}}=\left(\partial_{y_{k}} h\right)_{T, \rho, y_{l}}-\left(\partial_{\rho} h\right)_{T, y_{l}} \frac{\left(\partial_{\mathrm{y}_{k}} p^{0}\right)_{T, \rho, \mathrm{y}_{l}}}{\left(\partial_{\rho} p^{0}\right)_{T, \mathrm{y}_{l}}}
$$

so that at a mechanical thermodynamic unstable point where $\left(\partial_{\rho} p^{0}\right)_{T, y_{l}}=0$ the species specific enthalpies $h_{k}, k \in \mathfrak{S}$, are exploding and thus also some components of the matrix $L$. This is an expected behavior since various singularities in the transport coefficients may also arise at critical points [112, 113]. In order to illustrate this behavior, Figure 13 presents the $\mathrm{H}_{2}$ enthalpy as function of the normal coordinate $y$ in a $\mathrm{O}_{2}-\mathrm{H}_{2}$ strained diffusion flames with an ambient pressure of 51, 70 and 90 bars. Since the minimum value of the derivative $\left(\partial_{\rho} p^{0}\right)_{T, y_{l}}$ is gradually decreasing towards zero near the pseudo vaporizing zone, when the ambient pressure is decreased towards the oxygen critical pressure $p_{c}=50.43 \mathrm{bar}$, the corresponding specific enthalpy of hydrogen $h_{\mathrm{H}_{2}}(y)$ is gradually exploding. Note incidentally that, in constrast, the species Gibbs functions always remain smooth since $\left(\partial_{\rho} g\right)_{T, y_{l}}$ is proportional to $\left(\partial_{\rho} p^{0}\right)_{T, y_{l}}$.

It is therefore necessary to control the size of the vector $h$ making use of the fact that in the cold part of an oxygen/hydrogen diffusion flame there is mainly oxygen. To this aim, the derivative $\left(\partial_{\mathrm{y}_{k}} p^{0}\right)_{T, \rho, y_{l}}$ is rewritten in the form

$$
\left(\partial_{\mathrm{y}_{k}} p^{0}\right)_{T, \rho, \mathrm{y}_{l}}=\frac{\rho m}{m_{k}}\left(\partial_{\rho} p^{0}\right)_{T, \mathrm{y}_{l}}+\mathcal{R}_{k},
$$

where the residual $\mathcal{R}_{k}$ is given by

$$
\mathcal{R}_{k}=\left\{\frac{R T}{(v-b)^{2}}+\frac{m a}{v(v+b)^{2}}\right\} \sum_{j \in \mathfrak{S}} \mathrm{y}_{j}\left(\frac{b_{k}}{m_{j}}-\frac{b_{j}}{m_{k}}\right)+\frac{2 m a}{v(v+b)} \sum_{j, l \in \mathfrak{S}} \mathrm{y}_{j} \mathrm{y}_{l} \alpha_{l}\left(\frac{\alpha_{j}}{m_{k}}-\frac{\alpha_{k}}{m_{j}}\right) .
$$

The interest of this formulation (74) is that $\mathcal{R}_{k}$ vanishes for the pure species state $\mathrm{y}_{k}=1$ and $\mathrm{y}_{l}=0$, $l \in \mathfrak{S}, l \neq k$. The species enthalpies in (71)(72) are then written in the modified form $\widetilde{h}_{k}, k \in \mathfrak{S}$, with

$$
\widetilde{h}_{k}=\left(\partial_{\mathrm{y}_{k}} h\right)_{T, \rho, \mathrm{y}_{l}}-\frac{\rho m}{m_{k}}\left(\partial_{\rho} h\right)_{T, \mathrm{y}_{l}}-\frac{\mathcal{R}_{k}}{\mathfrak{f}\left(\left(\partial_{\rho} p^{0}\right)_{T, y_{l}}\right)}\left(\partial_{\rho} h\right)_{T, y_{l}},
$$

where $\mathfrak{f}$ is a smooth approximation of the function $\mathfrak{f}(x)=\max (\mathrm{m}, x)$ for a positive constant $\mathrm{m}>0$ which depends on the mixture upon consideration. These modified enthalpies are such that $\widetilde{h}_{k}=h_{k}$ away from the vaporizing zone as well as when the $k$ th species is the only present and remains always bounded. For a $\mathrm{H}_{2}-\mathrm{O}_{2}$ diffusion flame, the points where the matrix $L$ is effectively modified only concern zones of the flames where there is essentially only oxygen and where temperature is also nearly constant so that the stabilization of the matrix $L$ does not significantly modify the physics involved. 
For similar reasons we did not include in the model the complex behavior of transport coefficients near critical points.

In addition, with the traditional form of dissipative fluxes (21)(22) involving the diffusion driving forces (18), various singularities arise at mechanical thermodynamic stability limits. Indeed, since pressure is held constant in the partial derivations of the coefficients in (18), the species volume per unit mass $v_{j}, j \in \mathfrak{S}$, the thermodynamic coefficients $\Gamma_{i j}, i, j \in \mathfrak{S}$, are exploding when $\partial_{\rho} p^{0}=0$ like the species enthalpies per unit mass $h_{j}, j \in \mathfrak{S}$. Therefore, the traditional formulation of transport fluxes (21) - (22) is not recommended and the thermodynamical formulation (13)(14) is mandatory.

\subsection{A strained low-Mach diffuse-interface flame model}

Proceeding as for classical fluids in the low Mach number limit [52] we expand the bulk thermodynamic pressure as $p^{0}=\bar{p}^{0}+\widetilde{p}^{0}$ with $\bar{p}^{0}$ denoting the zeroth order bulk pressure and $\widetilde{p}^{0}$ the fluid dynamic perturbation. Bulk thermodynamic properties may thus be evaluated at the zeroth order pressure $\bar{p}^{0}$. The zeroth order pressure tensor then reads $\overline{\mathcal{P}}=\bar{p}^{0} \boldsymbol{I}-\frac{1}{2} \kappa|\nabla \rho|^{2} \boldsymbol{I}+\kappa \boldsymbol{\nabla} \rho \otimes \boldsymbol{\nabla} \rho-\kappa \rho \Delta \rho \boldsymbol{I}$ and the momentum equation yields that $\boldsymbol{\nabla} \cdot \overline{\mathcal{P}}=0$. Assuming now that the interface is planar with all quantities only depending spatially on a normal coordinate $y$, the normal component of the zeroth order momentum equation reads

$$
p^{\infty}=\bar{p}^{0}+\frac{1}{2} \kappa\left(\partial_{y} \rho\right)^{2}-\kappa \rho \partial_{y}^{2} \rho,
$$

where $p^{\infty}$ denotes the constant pressure in the bulk phases far from the interface. and the resulting zeroth order pressure tensor is in the form $\overline{\mathcal{P}}=\left(p^{\infty}-\kappa\left(\partial_{y} \rho\right)^{2}\right)\left(\boldsymbol{I}-\boldsymbol{e}_{y} \otimes \boldsymbol{e}_{y}\right)+p^{\infty} \boldsymbol{e}_{y} \otimes \boldsymbol{e}_{y}$ where $\boldsymbol{e}_{y}$ denotes the unit vector normal to the interface, so that normal to the interface the effective pressure is constant equal to $p^{\infty}$ whereas there are attractive forces tangential to the interface and the corresponding energy $\int_{-\infty}^{\infty} \kappa\left(\partial_{y} \rho\right)^{2} \mathrm{~d} y$ is interpreted as surface tension [100,101].

For a self similar structure analogous to that used in the previous section, the steady equation are found in the form

$$
\begin{aligned}
\rho \widetilde{u}+\partial_{y}(\rho v) & =0, \\
\rho v \partial_{y} \mathrm{y}_{i}+\partial_{y} \mathcal{J}_{i} & =m_{i} \omega_{i}, \quad i \in \mathfrak{S}, \\
\rho \widetilde{u}^{2}+\rho v \partial_{y} \widetilde{u}-J+\partial_{y}\left(\eta \partial_{y} \widetilde{u}\right) & =0, \\
\rho v \partial_{y} h-v \partial_{y} \bar{p}^{0}+\partial_{y}\left(\kappa \rho\left(\widetilde{u}+\partial_{y} v\right) \partial_{y} \rho\right)+\partial_{y} \mathcal{J}_{\mathrm{e}} & =0,
\end{aligned}
$$

with similar boundary conditions (52)(53) at both ends $\pm \infty$ and the normal velocity is centered at the origin (56).

\subsection{Transcritical $\mathrm{O}_{2}-\mathrm{H}_{2}$ flames}

The numerical simulations concern high pressure $\mathrm{O}_{2}-\mathrm{H}_{2}$ diffusion flames where $\mathrm{O}_{2}$ is injected at $100 \mathrm{~K}$. A detailed analysis of the thermodynamic stability of $\mathrm{O}_{2}-\mathrm{H}_{2} \mathrm{O}$ mixtures has first been conducted in order to understand $\mathrm{O}_{2}$ vaporizing interfaces in the presence of $\mathrm{H}_{2} \mathrm{O}$. Numerical simulations indeed show that in $\mathrm{O}_{2} / \mathrm{H}_{2}$ diffusion flames mainly $\mathrm{H}_{2} \mathrm{O}$ is diffusing from the flame front towards the incoming cold oxygen. The other species either disappear in the flame front or are too unstable to survive the cold temperature near the $\mathrm{O}_{2}$ vaporizing or pseudo-vaporizing interface. There is an exception for the species $\mathrm{H}_{2} \mathrm{O}_{2}$ which is metastable but which is only present in trace amount near oxygen vaporizing interfaces and has not been considered for the benefit of simplicity.

Following the classification of Van Konynenburg and R.L. Scott [114, 115], the liquid/vapor binary phase diagram for $\mathrm{O}_{2}-\mathrm{H}_{2} \mathrm{O}$ is of type III and very good agreement has been obtained between numerical simulations using the SRK equation of state and experimental measurements of Japas and Frank [116, 7]. The $\mathrm{O}_{2}-\mathrm{H}_{2} \mathrm{O}$ stability diagram shows that it is necessary to take into account the condensation of water below a pressure of about eighty bar. The species of the new phase to be added is thus $\mathrm{H}_{2} \mathrm{O}(l)$ - assuming that the local pressure remains below the critical pressure of water 220.6 bar-and the vaporization/condensation reaction is in the form

$$
\mathrm{H}_{2} \mathrm{O} \rightleftarrows \mathrm{H}_{2} \mathrm{O}(l)
$$

where we have neglected trace amounts of diluted species in $\mathrm{H}_{2} \mathrm{O}(l)$. Water in then present in two different phases, i.e., as a liquid as well as a species of the gas-like phase. Such droplets have notably 
been observed experimentally by Powell using liquid nitrogen [117]. Such a condensation phenomenon is similar mutatis mutandis to that observed when injecting alkane or alkene in nitrogen at high pressure $[111,118]$. In a flame structure, the liquid water is due to the condensation of water coming from the flame front by diffusion that cannot enter further into liquid oxygen since these fluids are largely immiscible at low temperature. These droplets are only present near the interface and their size has been discussed by Lafon et al. [110]. The droplets are sufficiently small in such a way that they follow the fluid local temperature and velocity [110]. The liquid water governing equation is in the form

$$
\partial_{t} \rho_{n+1}+\nabla \cdot\left(\rho_{n+1} \boldsymbol{v}\right)=m_{n+1} \omega_{n+1}
$$

where $n+1$ is the $\mathrm{H}_{2} \mathrm{O}(l)$ species index. The source term is modeled according to statistical mechanics $[50,51,31]$ and approximated in the form

$$
\omega_{n+1} \simeq \mathcal{K}_{\star}\left(g_{\mathrm{H}_{2} \mathrm{O}}^{0}-g_{\mathrm{H}_{2} \mathrm{O}(l)}^{0}\right)
$$

since the interface zone is nearly isotherm and with $\mathcal{K}_{\star}=\mathcal{K}_{\star}^{\prime} \mathrm{y}_{\mathrm{H}_{2} \mathrm{O}(j)}$ where $\mathcal{K}_{\star}^{\prime}$ is a constant and $j$ is the gaseous phase when condensation dominates whereas $j$ is the liquid phase when vaporization dominates. Such a simplified model of the liquid water dispersed phase has already been used by Lafon at al. [110] when investigating water condensation around isolated Lox droplets.

We present typical steady state $\mathrm{O}_{2}-\mathrm{H}_{2}$ diffusion flame structures in the subcritical and supercritical domains. These flames are obtained by flowing pure liquid like oxygen towards pure gaseous like hydrogen. We consider a subcritical pressure of $p^{\infty}=45$ bar and a supercritical pressure of $p^{\infty}=$ 60 bar - with respect to the oxygen critical pressure of $p_{c}=50.43$ bar. The temperature in the $\mathrm{O}_{2}$ cold stream is $T_{-}=100 \mathrm{~K}$ and in the $\mathrm{H}_{2}$ hot stream $T_{+}=300 \mathrm{~K}$. In all simulations, we have used a reaction mechanism of $O$ Conaire et al. and a value of $\kappa=10^{-2} \mathrm{~g}^{-1} \mathrm{~cm}^{7} \mathrm{~s}^{-2}$ of the capillary parameter and $\mathcal{K}_{\star}^{\prime}=10^{-2} \mathrm{~mol} \mathrm{~s} \mathrm{~cm}{ }^{-5}$ of the vaporization/condensation reaction parameter.

Figure 14 and Figure 15 present the temperature $T$ and species mole fractions $x_{i}, i \in \mathfrak{S}$, as functions of the normal coordinate $y$ in the diffusion flame structure at $p^{\infty}=60$ bar and $p^{\infty}=45$ bar respectively. The temperature curve is bell-shaped as typical of diffusion flames. Since the pressures are either $p^{\infty}=60$ bar or $p^{\infty}=45$ bar the flame structures are much thinner than at atmospheric pressure and the role of of the $\mathrm{HO}_{2}$ and $\mathrm{H}_{2} \mathrm{O}_{2}$ radicals is typical of high pressure flames hydrogen flames. The species mole fractions profiles $\mathrm{x}_{i}(y), i \in \mathfrak{S}$, presented in Figure 14 and Figure 15 also include liquid water that is localized near the (pseudo-)vaporization interface. In this pseudo-vaporization zones, when the pressure is subcritical, the local thermodynamic pressure $\bar{p}^{0}$ essentially follows an isotherm path of the SRK equation of state [7]. Finally, the diffuse interface transcritical flame model is able to smoothly transition from subcritical to supercritical conditions.

\section{Conclusion}

The impact of high pressure multicomponent transport models on the structure of supercritical flames has been investigated. Nonidealities have been shown to be important in cold zones of mixing layers and flames especially near thermodynamic stability. The thermodynamic form of transport fluxes has also been found mandatory for all pressure models in the presence of mechanical stability limits. Finally, a complete satisfactory kinetic theory of mixtures of dense gases is still missing and accurate high pressure correlations for Soret coefficients as well as volume viscosities would also be welcome.

\section{References}

[1] M. El Gamal, E. Gutheil and J. Warnatz, The structure of laminar premixed $H_{2}$-Air flames at elevated pressures, Zeit. Phys. Chem. 214 (2000), pp. 419-435.

[2] N. A. Okongo and J. Bellan, Direct numerical simulation of a transitional supercritical binary mixing layer: heptane and nitrogen, J. Fluid Mech. 464 (2002), pp. 1-34.

[3] G. Ribert, N. Zong, V. Yang, L. Pons, N. Darabiha, and S. Candel, Counterflow diffusion flames of general fluids: Oxygen/Hydrogen mixtures, Comb. Flame 154 (2008), pp. 319-330.

[4] L. Pons, N. Darabiha, S. Candel, G. Ribert, and V. Yang, Mass transfer and combustion in transcritical non-premixed counterflows, Comb. Theory Mod. 13 (2009), pp. 57-81. 
[5] V. Giovangigli, L. Matuszewski, and F. Dupoirieux, Detailed Modeling of Transcritical Planar $\mathrm{H}_{2}$ $\mathrm{O}_{2}-\mathrm{N}_{2}$ Flames, Comb. Theory Mod. 15 (2011), pp. 141-182.

[6] V. Giovangigli and L. Matuszewski, Numerical Simulation of Transcritical Strained Laminar Flames, Combustion and Flame, 159 (2012), pp. 2829-2840.

[7] P. Gaillard, V. Giovangigli, and L. Matuszewski, A Transcritical Diffuse Interface H2/LOX flame model, Comb. Theory Mod. 20 (2016), pp. 486-520.

[8] P. Gaillard, V. Giovangigli, and L. Matuszewski, Nonmixing layers, Physical Review Fluids, 1, (2016), 084001.

[9] H. Nomura, S. Nakaya, and M. Tsue, Microgravity research on quasi-steady and unsteady combustion of fuel droplet at high pressures, in "High Pressure Flows for Propulsion Applications", edited by J. Bellan, Vol. ???, AIAA Progress Series, (2018), pp. ???-???.

[10] J.C. Oefelein, Thermophysical characteristics of shear-coaxial $\mathrm{LOX}-\mathrm{H}_{2}$ flames at supercritical pressure, Proc. Comb. Inst. 30 (2005), pp. 2929-2937.

[11] J. Bellan, Theory, modeling and analysis of turbulent supercritical mixing, Comb. Sci. Tech. 178 (2006), pp. 253-281.

[12] N. Zong and V. Yang, Near-field flow and flame dynamics of LOX/methane shear-coaxial injector under supercritical conditions, Proc. Comb. Inst. 30 (2007), pp. 2309-2317.

[13] T. Schmitt, Y. Méry, M. Boileau, and S. Candel, Large-Eddy Simulation of Oxygen/Methane Flames under Transcritical Conditions, Proc. Comb. Inst. 33 (2011), pp. 1383-1390.

[14] A. Gnanaskandan and J. Bellan, Large Eddy Simulations of high pressure jets: Effect of subgrid scale modeling, in "High Pressure Flows for Propulsion Applications", edited by J. Bellan, Vol. ???, AIAA Progress Series, (2018), pp. ???-???.

[15] S. Matsuyama, H. Terashima, M. Koshi, and A. Umemura, Detailed modeling of supercritical jets and flames, in "High Pressure Flows for Propulsion Applications", edited by J. Bellan, Vol. ???, AIAA Progress Series, (2018), pp. ???-???.

[16] G. Ribert, P. Domingo, X. Petit, N. Vallée, J.-B. Blaisot, Modelling and simulations of highpressure practical flows, in "High Pressure Flows for Propulsion Applications", edited by J. Bellan, Vol. ???, AIAA Progress Series, (2018), pp. ???-???.

[17] Z. Yue and R. D. Reitz, Simulation of the high pressure combustion process in diesel engines, in "High Pressure Flows for Propulsion Applications", edited by J. Bellan, Vol. ???, AIAA Progress Series, (2018), pp. ???-???.

[18] M. Habiballah, M. Orain, F. Grisch, L. Vingert, and P. Gicquel, Experimental studies of high pressure cryogenic flames on the MASCOTTE facility, Comb., Sci. Tech. 178 (2006), pp. 101-128.

[19] S. Candel, M. Juniper, G. Singla, P. Scouflaire, and C. Rolon, Structure and dynamics of cryogenic flames at supercritical pressure, Comb. Sci. Tech. 178 (2006), pp. 161-192.

[20] S. Sardeshmukh, C. Huang, R. Gejji, M. Bedard, Z. Hallum, R. Lucht, and W. Anderson, Highpressure experiments relevant to rocket propulsion, in "High Pressure Flows for Propulsion Applications", edited by J. Bellan, Vol. ???, AIAA Progress Series, (2018), pp. ???-???.

[21] M. Oschwald, D. Suslov, J. Haemisch, O. Haidn, M. Celano, C. Kirchberger, N. Rackemann, A. Preuß and D. Wiedmann, Measurement of Heat Transfer in Liquid Rocket Combustors, in "High Pressure Flows for Propulsion Applications", edited by J. Bellan, Vol. ???, AIAA Progress Series, (2018), pp. ???-???.

[22] J. Meixner, Zur Thermodynamik der Thermodiffusion, Ann. der Phys., 41 (1941), pp. 333-356.

[23] J. Meixner, Zur Thermodynamik der irreversiblen Prozesse in Gasen mit chemisch reagierenden, dissoziierenden und anregbaren Komponenten, Ann. der Phys., 43 (1943), pp. 244-270.

[24] I. Prigogine, Etude thermodynamique des phénomènes irréversibles, Dunod, Paris, (1947). 
[25] L. Onsager, Reciprocal relations in irreversible processes, Phys. Rev., 37 (1931), pp. $405-426$.

[26] S.R. de Groot and P. Mazur, Non-Equilibrium Thermodynamics, Dover publications, Inc. New York, 1984.

[27] C. Eckart, The thermodynamic of irreversible processes, II. Fluid mixtures, Phys. Rev., 58 (1940), pp. 269-275.

[28] J. H. Irving and J. G. Kirkwood, The statistical mechanics of transport processes. IV. The equations of hydrodynamics, J. Chem. Phys., 18 (1950), pp. 817-829.

[29] R. J. Bearman and J. G. Kirkwood, The statistical mechanics of transport processes. XI. Equations of transport in multicomponent systems, J. Chem. Phys., 28 (1958), pp. 136-145.

[30] H. Mori, Statistical-mechanical theory of transport in fluids, Phys. Rev., 112 (1958), pp. 1829-1842.

[31] J. Keizer, Statistical Thermodynamics of Nonequilibrium Processes, Springer Verlag, New York (1987)

[32] L. Waldmann, Transporterscheinungen in Gasen von mittlerem Druck, Handbuch der Physik, 12 , (1958) 295-514.

[33] J. Van de Ree, On the definition of the diffusion coefficients in reacting gases, Physica, 36 (1967), pp. $118-126$.

[34] S. Chapman and T.G. Cowling, The mathematical theory of non-uniform gases Cambridge University Press, Cambridge, 1970.

[35] J. H. Ferziger and H. G. Kaper, Mathematical theory of transport processes in gases North-Holland Publishing Company, Amsterdam, (1972).

[36] J. O. Hirschfelder, C. F. Curtiss, and R. B. Bird, Molecular theory of gases and liquids, Wiley, New York (1954).

[37] A. Ern and V. Giovangigli, Multicomponent transport algorithms Lecture Notes in Physics, Monograph m24, Springer-Verlag, Heidelberg, (1994).

[38] L. Bajaras, L.S. Garcia-Colin, and E. Piña, On the Enskog-Thorne Theory for a binary mixture of dissimilar rigid spheres, J. Stat. Phys., 7 (1973), pp. 161-183.

[39] H. Van Beijeren and M. H. Ernst, The Modified Enskog Equations, Phys. A 68 (1973), pp. 437-456.

[40] H. Van Beijeren and M. H. Ernst, The Modified Enskog Equations for Mixtures, Phys. A 70 (1973), pp. 225-242.

[41] V. I. Kurochkin, S. F. Makarenko, and G. A. Tirskii, Transport coefficients and the Onsager relations in the kinetic theroy of dense gas mixtures, J. Appl. Mech. Tech. Phys. 25 (1984), pp. $218-225$.

[42] V. Giovangigli and L. Matuszewski, Supercritical fluid thermodynamics from equations of state, Physica D 241 (2012), 649-670.

[43] O. Redlich and J. N. S. Kwong, On the thermodynamics of solutions. $V$ An equation of state. Fugacities of gaseous solutions, Chem. Reviews 44 (1949), pp. 233-244.

[44] G.S. Soave, Equilibrium constants from a modified Redlich-Kwong equation of state, Chem. Eng. Sci. 27 (1972), pp. 157-172.

[45] A. Congiunti, C. Bruno, and E. Giacomazzi, Supercritical combustion properties, in 11th Aerospace Sciences Meeting and Exhibit, Reno, Nevada, USA, 6-9 January, AIAA-2003-478, 2003.

[46] P. Colonna and P. Silva, Dense gas thermodynamic properties of single and multicomponent fluids for fluid dynamics simulations, J. Fluid Eng. 125 (2003), pp. 414-427. 
[47] W.A. Cañas-Marín, U.E. Guerrero-Aconcha, and J.D. Ortiz-Arango, Comparison of different cubic equations of state and combination rules for predicting residual chemical potential of binary and ternary Lennard-Jones mixtures, Fluid Phase Equil. 234 (2005), pp. 42-50.

[48] W.A. Cañas-Marín, J.D. Ortiz-Arango, U.E. Guerrero-Aconcha, and C.P. Soto-Tavera, Thermodynamic derivative properties and densities for hyperbaric gas condensates: SRK equation of state predictions versus Monte Carlo data, Fluid Phase Equil. 253 (2007), pp. 147-154.

[49] R.C. Reid, J.M. Prausnitz, B.E. Poling, The Properties of Gases and Liquids McGraw-Hill, NewYork, (1987).

[50] M. R. Marcelin, Sur la Mécanique des Phénomènes Irréversibles, Comptes Rendus de l'Académie des Sciences de Paris, Séance du 5 décembre 1910, (1910), pp. 1052-1055.

[51] M. R. Marcelin, Contribution à l'étude de la cinétique physico-chimique, Thèses de la Faculté des Sciences de Paris, (1914).

[52] V. Giovangigli, Multicomponent flow modeling, Birkhaüser, Boston, (1999).

[53] T.H. Chung, M. Ajlan, L.L. Lee, and K.E. Starling, Generalized Multiparameter correlation for nonpolar and polar fluid transport properties, Ind. Eng. Chem. Res., 27 (1988), pp. 671-679.

[54] J.F. Ely and H.J. Hanley, Prediction of transport properties. 2. Thermal conductivity of pure fluids and mixtures, Indus. Eng. Chem. Fund. 22 (1983), pp. 90-97.

[55] V. Giovangigli and L. Matuszewski, Mathematical Modeling of Supercritical Multicomponent Reactive Fluids, Math. Mod. Meth. App. Sci., 23, (2013), pp. 2193-2251.

[56] R. Taylor and R. Krishna, Multicomponent mass transfer, John Wiley, New York, (1993).

[57] K.G. Harstad and J. Bellan, Mixing rules for multicomponent mixture mass diffusion coefficients and thermal diffusion factors, J. Chem. Phys. 120-12 (2004), pp. 5664-5673.

[58] V. M. Zhdanov, Transport processes in multicomponent plasmas, Taylor and Francis, London, (2002).

[59] W. A. Wakeham, The binary diffusion coefficient of moderately dense gas mixtures, J. Phys. B., 6 (1973), pp. 372-383.

[60] S. Takahashi, Preparation of a generalized chart for the diffusion coefficients of gases at high pressures, J. Chem. Eng. Jap., 7 (1974), pp. 417-420 .

[61] K. Harstad, and J. Bellan, High pressure binary mass diffusion coefficients for combustion applications, Ind. Eng. Chem. Res., 43 (2004), pp. 645-654.

[62] A. Leahy-Dios and A. Firoozbadi, Unified model for nonideal multicomponent molecular diffusion coefficients, AIChE J., 53 (2007), pp. 2932-2939 .

[63] V. Giovangigli, Convergent iterative methods for multicomponent diffusion, Impact Comput. Sci. Eng., 3 (1991), pp. 244-276.

[64] A. Ern and V. Giovangigli, Projected iterative algorithms with application to multicomponent transport, Linear Algebra Appl., 250 (1997), pp. 289-315.

[65] A. Ern and V. Giovangigli, Optimized transport algorithms for flame codes, Comb. Sci. Tech., 118 (1996), pp. 387-395.

[66] V. Giovangigli, Multicomponent transport algorithms for partially ionized plasmas, J. Comp. Phys., 229 (2010), pp. 4117-4142.

[67] E. S. Oran and J. P. Boris, Numerical simulation of reactive flows Cambridge university press, Cambridge, (2001).

[68] A. Ern and V. Giovangigli, Thermal diffusion effects in hydrogen-air and methane-air flames, Comb. Theory Mod. 2-4 (1998), pp. 349-372. 
[69] M. D. Smooke, Solution of burner stabilized premixed laminar flames by boundary value methods, J. Comp. Phys. 48 (1982), pp. 72-105.

[70] M.D. Smooke, The Computation of Laminar Flames, Proc. Comb. Inst. 34 (2013) 65-98.

[71] V. Giovangigli and M. D. Smooke, Adaptive continuation algorithms with application to combustion problems, Appl. Numer. Math., 5 (1989), pp. 305-331

[72] V. Giovangigli V and N. Darabiha, Vector Computers and Complex Chemistry Combustion, Mathematical Modeling in Combustion and Related Topics (Brauner C and Schmidt-Laine C Eds. Nijhoff M Pug.) NATO ASI Series, 140 (1988), pp. 491-503.

[73] R. Bendakhlia, V. Giovangigli, and D. Rosner, Soret effects in laminar counterflow spray diffusion flames, Comb. Theory Mod., 6 (2002), pp. 1-17.

[74] A. Ern and V. Giovangigli, EGlib server and User's manual, http://www.cmap.polytechnique.fr/www.eglib.

[75] L. Matuszewski, PhD Thesis, University Paris 6, 2011.

[76] M. Pertler, E. Blass, and G.W. Stevens, Fickian Diffusion in Binary Mixtures that Form Two Liquid Phases, AIChE J., 42 (1996), pp. 910-920.

[77] E.L. Cussler, Diffusion, Mass Transfer in Fluid Systems, Cambridge, (2009).

[78] A.G. Lamorgese, D. Molin and R. Mauri, Phase Field Approach to Multiphase Flow Modeling, Milan J. Math., 79 (2011), pp. 597-642.

[79] R. Mauri, Non-Equilibrium Thermodynamics in Multiphase Flows, Springer, 2013.

[80] L. Ting, On the Mixing of Two Parallel Streams, J. Math. Phys. 28, (1959), pp. 153-165.

[81] C.A. Kennedy and T.B. Gatski, Self-Similar Supersonic Varaible Density Shear Layers in Binary Systems, Phys. Fluids 6, (1994), pp. 662-673.

[82] T. T. H. Verschoyle, The ternary system Monoxyde-Nitrogen-Hydrogen and the component binary systems between temperatures of -185 degrees and -215 degrees $C$., and between pressures of 0 and 225 atm, Phil. Trans. R. Soc. Lond., A230 (1932), pp. 189-220.

[83] L. S. Eubanks, Vapor-Liquid equilibria in the system Hydrogen-Nitrogen-Carbon monoxide, The Rice Institute, Houston, Texas, (1957).

[84] M. Sermange, Mathematical and numerical aspects of one-dimensional laminar flame simulation, Appl. Math. Opt., 14 (1986), pp. 131-153.

[85] V. Giovangigli and M. Smooke, Application of continuation techniques to plane premixed laminar flames, Comb. Sci. and Tech., 87 (1992), p 241-256.

[86] J. Warnatz, Survey of rate coefficients in the $C / H / O$ system, in : W. C. Gardiner Ed., Combustion Chemistry, Springer, New York (1984), 197-360.

[87] F. Behrendt and J. Warnatz, The dependence of flame propagation in $\mathrm{H}_{2}-\mathrm{O}_{2}-\mathrm{N}_{2}$ mixtures on temperature, pressure and initial composition, Int. J. Hydrogen Energ., 10 (1985), pp. 749-755.

[88] J. Warnatz, U. Mass, and R.W. Dibble, Combustion, Springer, (2006).

[89] M Ó Conaire, H. J. Curran, J. M. Simmie, W. J. Pitz, and C. K. Westbrook, A comprehensive modeling study of Hydrogen oxydation, Int. J. Chem. Kin., 36 (2004), pp. 603-622.

[90] A.T. Holley, X.Q. You, E. Dames, H. Wang, F.N. Egolfopoulos, Sensitivity of propagation and extinction of large hydrocarbon flames to fuel diffusion, Proc. Comb. Inst. 32 (2009), pp. 1157-1163

[91] S. Jay, F. Lacas and S. Candel, Combined surface density concepts for dense spray combustion, Comb. and Flame 144 (2006) 558-577. 
[92] J.D. van der Waals, Thermodynamische Theorie der Capillariteit in de Onderstelling van Continue Dichtheidsverandering Verhand. Kon. Akad. V Wetensch. Amst., (1893). (in Dutch; English translation in J. Stat. Phys., 20 (1979), pp. 197-244).

[93] J. D. van der Waals, Thermodynamisch Theorie der Kapillariät unter Voraussetzung stetiger Dichteanderung, Z. Phys. Chem., 13 (1894), pp. 657-725.

[94] Korteweg, D.J., Sur la Forme que Prennent les Equations du Mouvement Fluide si l'on tient Compte de Forces Capillaires Causées par les Variations de Densité Considérables mais Continues et sur la Théorie de la Capillarité dans l'Hypothèse d'une Variations Continue de la Densité, Arch. Neerl. Sci Exactes, 6 (1901), pp. 1-20.

[95] J. W. Cahn, and J. E. Hilliard, Free Energy of a Non Uniform System I, Interfacial Free Energy, J. Chem. Phys., 28 (1958), pp. 258-267.

[96] J. W. Cahn, and J. E. Hilliard, Free Energy of a Non Uniform System II, Thermodynamic Basis, J. Chem. Phys., 30 (1959), pp. 1121-1124.

[97] J.S. Rowlinson and B. Widom, Molecular Theory of Capillarity, Dover publications, Mineola, 2002.

[98] P. Seppecher, Moving Contact Line in the Cahn-Hilliard Theory, Int. J. Eng. Sci., 34 (1996), pp. 977-992.

[99] Anderson , D.M. and McFadden G.B. and Wheeler, A.A., Diffuse Interface Methods in Fluid Mechanics, Ann. Rev. Fluid Mech., 30 (1998), pp. 139-165.

[100] D. Jamet, O. Lebaigue, N. Coutris, and J. M. Delhaye, The Second Gradient Method for the Direct Numerical Simulation of Liquid-Vapor Flows with Phase Change, J. Comput. Phys., 169 (2001), pp. 624-651.

[101] D. Jamet, Diffuse interface models in fluid mechanics, pmc.polytechnique.fr/mp/GDR/docu/Jamet.pdf (2005).

[102] S. Benzoni-Gavage, R. Danchin, S. Descombes, and D. Jamet, Structure of Korteweg models and stability of diffuse interfaces, Interfaces and Free Bound. 7 (2005), pp. 371-414.

[103] H. Lin, Y.-Y. Duan, and Q. Min, Gradient Theory Modeling of Surface Tension for Pure Fluids and Binary Mixtures, Fluid Phase Equil., 254 (2007), pp. 75-90.

[104] J. Bellan, Supercritical (and subcritical) fluid behavior and modeling: drops, streams, shear and mixing layers, jets and sprays, Prog. En. Comb. Sci. 26 (2000), pp. 329-366.

[105] V. Yang, Modeling of Supercritical Vaporization, Mixing, and Combustion in Liquid-Fueled Propulsion Systems, Proc. Comb. Inst. 28 (2000), pp. 925-942.

[106] N. Zong and V. Yang, Near-field flow and flame dynamics of LOX/methane shear-coaxial injector under supercritical conditions, Proc. Comb. Inst. 30 (2007), pp. 2309-2317.

[107] J. C. Oefelein, R. N. Dahms, G. Lacaze, J.L. Manin, and L.M. Pickett, Effects of Pressure on the Fundamental Physics of Fuel Injection in Diesel Engines, ICLASS 2012 Conference, Heidelberg, (2012).

[108] R. N. Dahms and J. C. Oefelein, On the Transition between Two-Phase Interface Dynamics in Multicomponent Fluids at Supercritical Pressures, Physics of fluids, 25 (2013), pp. 1-24.

[109] P. Gaillard, PhD Thesis, University Paris 6, 2015.

[110] P. Lafon, H. Meng, V. Yang, and M. Habiballah, Vaporization of Liquid Oxygen (LOX) Droplets in Hydrogen and Water Envoronments under sub and super-Critical Conditions, Comb. Sci. Tech. 180 (2008), pp. 1-26.

[111] K.-C. Lin, C. Carter, X. Zuo and A. Sandy, Characterization of droplet nucleation inside supercritical ethylene jets using small-angle X-ray scattering (SAXS) technique, in "High Pressure Flows for Propulsion Applications", edited by J. Bellan, Vol. ???, AIAA Progress Series, (2018), pp. ???-???. 
[112] L.P. Kadanoff and J. Swift, Transport Coefficients near the Liquid-Gas Critical Point, Phys. Rev. 166 (1968), pp. 89-101.

[113] J.V. Sengers, Transport Processes Near the Critical Point of Gases and Binary Liquids in the Hydrodynamic Regime, Ber. Busen. Phys. Chem. 76 (1972), pp. 234-249.

[114] P.H. Van Konynenburg and R.L. Scott, Critical lines and Phase Equilibria in Binary Van Der Waals Mixtures, Phil. Trans. A, Royal Society, 298 (1980) pp. 495-540.

[115] J.M. Prausnitz, R.N. Lichtenthaler, and E.G. de Avezo, Molecular Thermodynamics of FluidPhase Equilibria, Prentica Hall, Upper saddle river, 1999.

[116] M.L. Japas and E.U. Franck, High Pressure Phase Equilibria and PVT-Data of the Water-Oxygen System Including Water-Air to $673 \mathrm{~K}$ and 250 MPa, Ber. Bunsenges. Phys. Chem. 89 (1985) pp. $1268-1275$.

[117] E.E. Powell, Simulation of Water Vapor Condensation on LOX Droplet Surface Using Liquid Nitrogen, NASA/ASEE Summer Faculty Fellowship Program, N89-21726 (1988), XXIII.

[118] L. Qiu and R. D. Reitz, An investigation of thermodynamic states during high-pressure fuel injection using equilibrium thermodynamics, International Journal of Multiphase Flow, 72 (2015) pp. 24-38. 